\title{
INVESTIGATION OF THE DIMER INTERFACE AND ACTIVE SITE IN PYRIDOXAL 5' PHOSPHATE ENZYMES CYSTATHIONINE $\beta$ - LYASE AND CYSTATHIONINE $\gamma$ - LYASE
}

\author{
By \\ Victoria Samakai, B.Sc. \\ A thesis submitted to the Faculty of Graduate and Postdoctoral Affairs in partial \\ fulfilment of the requirements for the degree of \\ Master of Science \\ in \\ Biology \\ Department of Biology \\ Ottawa-Carleton Institute of Biology \\ Carleton University \\ Ottawa, Ontario \\ December 2015
}

(C)2015

Victoria Samakai 


\section{Abstract}

Cystathionine $\beta$-lyase (CBL) catalyses the PLP-dependent $\beta$-elimination of cystathionine, yielding homocysteine, pyruvate and ammonia, while the $\gamma$-elimination of this pseudosymmetric substrate by cystathionine $\gamma$-lyase (CGL) produces cysteine, $\alpha$-ketobutyrate and ammonia. The distinct $\beta$ versus $\gamma$-elimination reaction specificity of the structurally conserved eCBL and yCGL enzymes provides an ideal system to probe the subtle factors that regulate activity and specificity. Studies exploring these factors will enable the continued development of our understanding of the mechanisms whereby PLP-dependent enzymes regulate substrate and reaction specificity, building a framework that will facilitate future studies aiming to engineer these enzymes to harness the power of this catalytically versatile cofactor for use in various industrial processes. Comparison of the structures of Escherichia coli CBL (eCBL) and Saccharomyces cerevisiae CGL (yCGL) identified differences at the dimer interface and within the active site. The effect of substitutions at these positions on enzyme properties is explored in chapters 2 and 3 of this thesis, respectively, as a precursor to studies probing their potential role as determinants of reaction specificity. Charge-reversal substitutions of the four residues involved in the K5-D382 and K17-D37 salt-bridges, which span the dimer interface of eCBL, were constructed and their effect on enzyme stability and activity determined. Single and double charge-reversal substitutions similarly reduce the catalytic efficiency by 11-21-fold, despite the greater effect of the former on the stability of the enzyme. This demonstrates that while even small conformational changes in this region are conveyed to the active site, the distinct dimer interfaces of eCBL and yCGL are unlikely to be a primary determinant of specificity. The potential of active-site residues S50 and S200 of yCGL to influence the positioning of the $\varepsilon$ amino group of the catalytic base was also explored, via characterization of the S50A, S50G and 
S200A substitutions of yCGL and the corresponding G57S and A207S of eCBL. The catalytic efficiency of these variants is reduced only 5-11-fold, compared to the respective wild-type enzymes, suggesting that these residues are not primary determinants in regulating the mobility of the catalytic base or in maintaining a catalytically competent active site architecture and likely do not regulate reaction specificity. 


\section{Statement of Contributions}

My contributions to the research described in this thesis include:

1. Development of the research questions and experimental design, in partnership with Dr. S.M. Aitken.

2. Responsibility for the collection and analysis of data.

3. The co-supervision, in collaboration with Dr. S.M. Aitken and Duale Ahmed (PhD candidate), of 1 undergraduate student: Hyung Yoo who participated in the construction, purification and steady-state characterization of site-directed variants in chapter 2.

4. The preparation of drafts of this thesis, which was edited under the guidance of Dr. S.M. Aitken.

I formally acknowledge the contributions of my supervisor, Dr. S.M. Aitken who contributed her expertise by guiding me in the formulation of the research questions and experimental design and by assisting with the analysis and interpretation of data and guidance in the writing of my thesis. Duale Ahmed constructed the primers for all 4 site-directed variants as well as purified and performed the preliminary characterization of 2 of the site-directed variants described in chapter 2. I have obtained permission from each of my co-authors to use collaborative works in this thesis. 


\section{Acknowledgements}

My journey as a Masters student at Carleton University has been an enlightening, educative and inspirational one, I thank God for allowing me this privilege. As behind every great student there is an even better teacher, special thanks go to my supervisor Dr. Susan M. Aitken, who was my guide through graduate school and a great guide at that. I appreciate her giving me the opportunity to work in her lab. The knowledge I've obtained through working with her has broadened my mindset in relation to science and improved the person I am as a whole. I would also like to extend my deepest thanks to my committee members, Dr. Alex Wong, Dr. Amanda. J. MacFarlane and Dr. Edana Cassol for the helpful feedback and comments I received from them.

To my fellow lab mates who imparted so much of their knowledge on me, Edgar Abouassaf, Duale Ahmed and Allison Jaworski, thank you for assisting me in times of confusion, I will always be grateful. I would also like to thank Hyung Yoo, an undergraduate student who I supervised for his honors thesis project that contributed to my thesis and taught me patience and understanding. Many thanks goes to my support system, Chantelle and Mwape Musambachime, Bobby Samakai, Elsie Samakai, Chilombo Maonde, Chileshe Chalikosa, Bwalya Musanya, Nkuka Chibesa, Chibuye Chola, Etambuyu Akapelwa and Precious Samakai, as well as all my other friends and family who were there to always lend an ear or shoulder. I would be amiss to not give my deepest thanks to my parents, Bob and Molly Samakai, who have encouraged me throughout my life to attain my goals and it is because of their belief in my abilities that I have been able to pursue and follow through with this degree. I will forever be grateful. 
Dedication

TO MY MUM AND DAD,

THANK YOU FOR ALL THAT YOU HAVE DONE AND CONTINUE TO DO 


\section{Table of Contents}

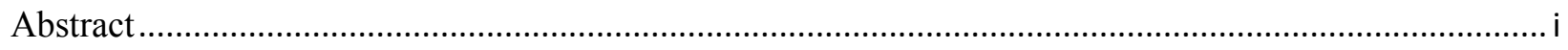

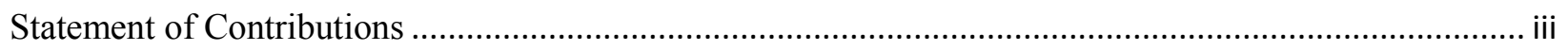

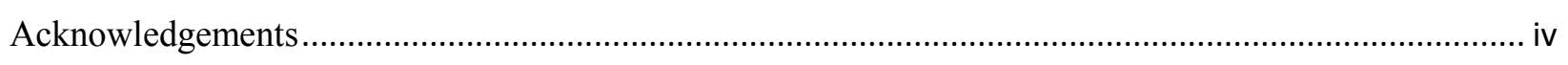

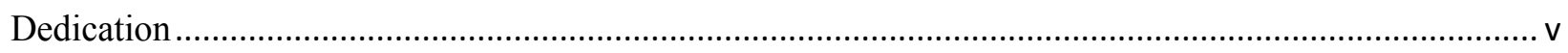

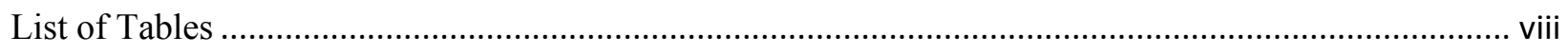

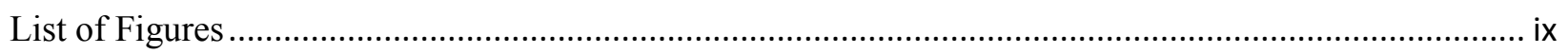

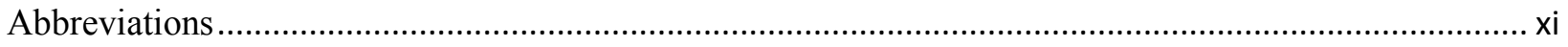

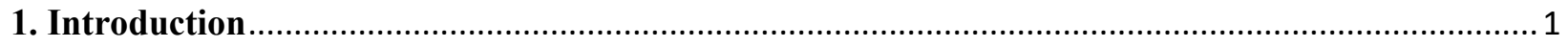

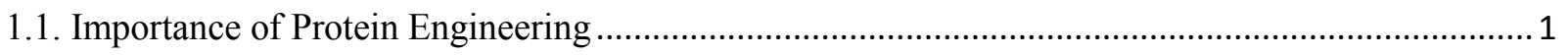

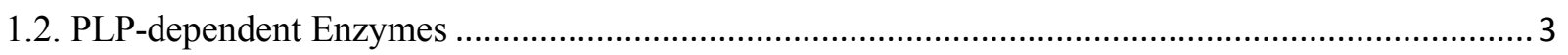

1.3. The Transsulfuration and Reverse Transsulfuration Pathways .................................................. 5

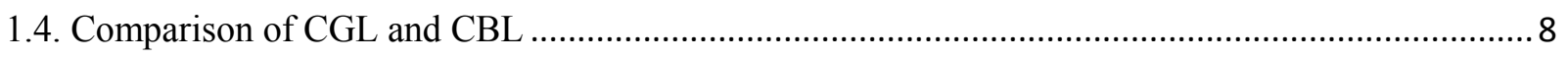

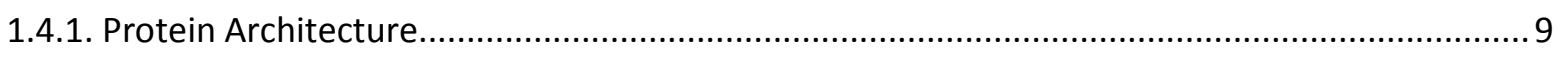

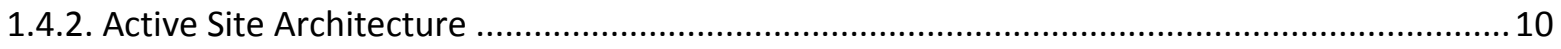

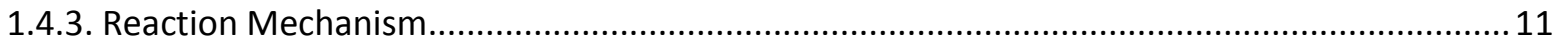

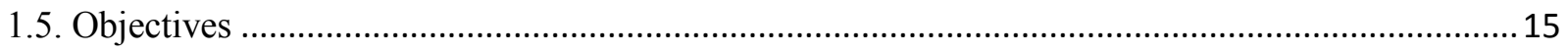

Chapter 2: Investigation of a pair of charged interactions at the dimer interface of cystathionine $\beta$ -

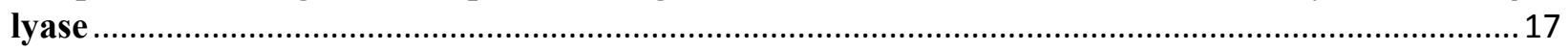

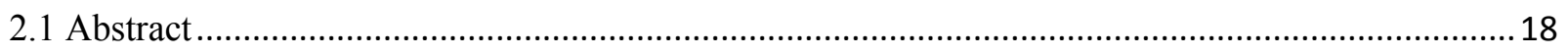

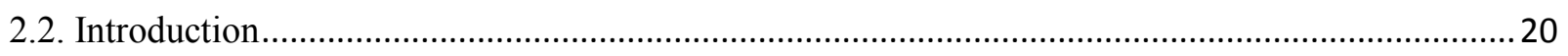

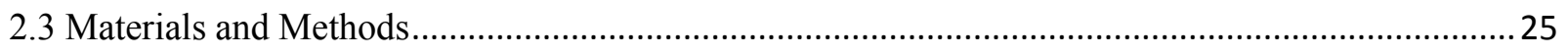

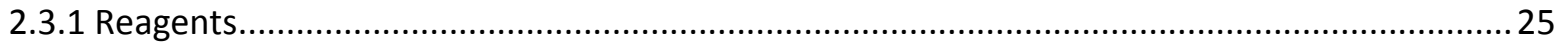

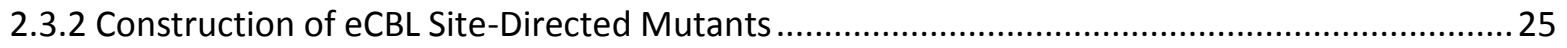

2.3.3 Expression and Purification of the Site-Directed Variants .....................................................2 27

2.3.4 Determination of Steady State Kinetic Parameters for the Hydrolysis of L-cystathionine ........28

2.3.5 Stability Studies of wt-eCBL and the Respective Site-Directed eCBL Variants .........................30

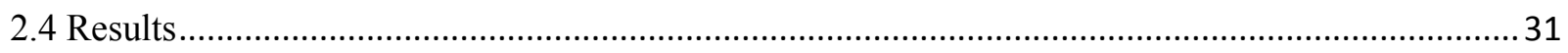

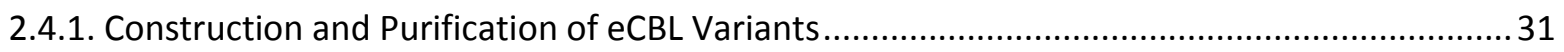


2.4.3. Urea Denaturation of wt-eCBL and eCBL Mutants ............................................................. 34

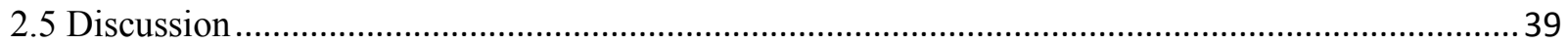

Chapter 3: Interconversion of a pair of residues in the active sites of cystathionine $\beta$-lyase and

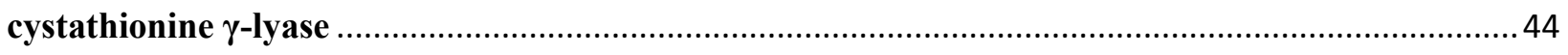

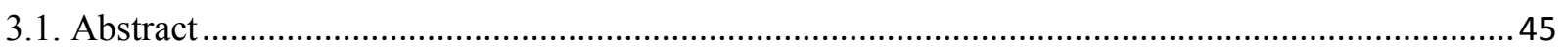

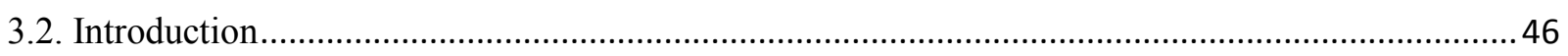

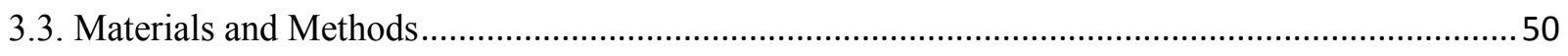

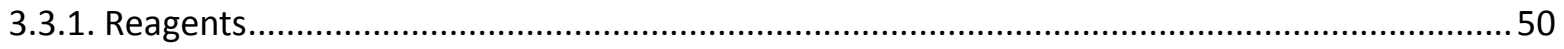

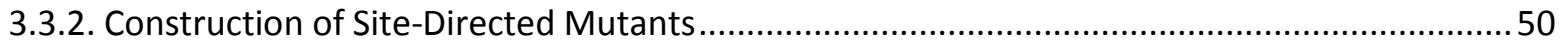

3.3.3. Expression and Purification of the Site-Directed Variants ....................................................53

3.3.4. Determination of Steady State Kinetic Parameters of the Hydrolysis of L-cystathionine ........54

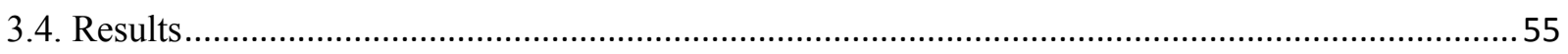

3.4.1. Construction and Purification of eCBL and yCGL Variants...................................................55

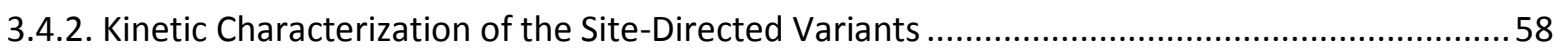

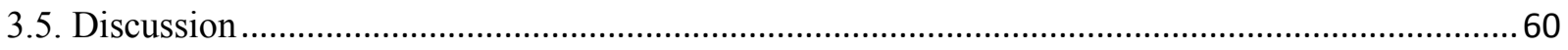

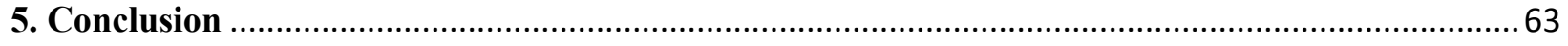

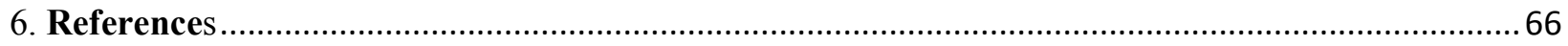




\section{List of Tables}

\section{Page}

Table 2.1. Sequences of the primers employed to construct the site-directed

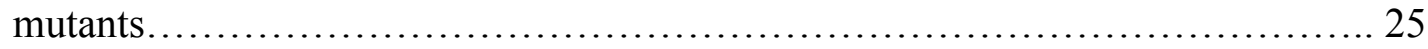

Table 2.2. Site directed variants and their protein yield .................................. 32

Table 2.3. Steady state kinetic parameters of wild-type eCBL and theK5D, K17D, D37K and D382K, K5D/D382K and K17D/D37K variants........................... 34

Table 3.1. Site-directed mutants and their respective mutagenic primers.................... 51

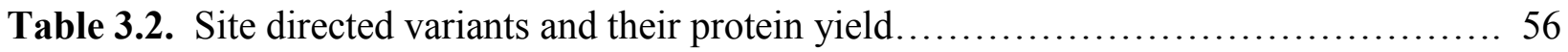

Table 3.3. Steady state kinetic parameters of wild-type and site-directed variants of yCGL and

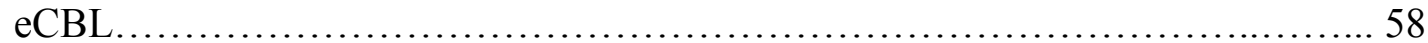




\section{List of Figures}

Figure 1.1. The transaldimination reaction, in which the $\alpha$-amino group of the substrate replaces the $\varepsilon$-amino group of the active-site lysine in Schiff base linkage with the cofactor is the first step in a range of PLP-catalyzed transformations of amino acid substrates

Figure 1.2. The bacterial/plant transsulfuration and mammalian/yeast reverse transsulfuration pathways which interconvert cysteine and homocysteine, the immediate precursor of methionine. Cystathionine is the common intermediate of both pathways... 7

Figure 1.3. The chemical mechanism of the $\gamma$-elimination of cystathionine catalyzed by CBL, to the point of the release of the cysteine product.

Figure 1.4. The chemical mechanism of the $\gamma$-elimination of cystathionine catalyzed by CGL, to the point of release of the homocysteine product...................... 14

Figure 2.1. The transulfuration pathway of bacteria showing the reactions catalyzed by cystathionine $\gamma$-synthase and cystathionine $\beta$-lyase.

Figure 2.2. A cartoon representation of the eCBL tetramer, distinguishing each subunit by colour.

Figure 2.3. The DTNB assay used to assay the L-cystathionine hydrolysis activity of eCBL

Figure 2.4. Agarose (1\%) gel of the 3' and 5' amplicons, labelled 'left rxn' and 'right rxn', for each site-directed mutant as well as the re-assembly products of the second PCR step in the construction of the site-directed mutants, via the overlap-extension method.

Figure 2.5. SDS-PAGE gel stained with Comassie Blue of the purification of eCBL-K5D

Figure 2.6. The dependence of the tryptophan emission spectrum of $0.5 \mu \mathrm{M}$ wt-eCBL on urea concentration

Figure 2.7. The dependence of the tryptophan emission spectrum of $0.5 \mu \mathrm{M}$ wild-type and K5D eCBL on urea concentration.

Figure 2.8. The dependence of the tryptophan emission spectrum of $0.5 \mu \mathrm{M}$ wild type and D37K eCBL on urea concentration.

Figure 2.9. The dependence of the tryptophan emission spectrum of $0.5 \mu \mathrm{M}$ wild type and $\mathrm{K} 5 \mathrm{D} / \mathrm{D} 382 \mathrm{~K}$ eCBL on urea concentration. 
Figure 2.10. The dependence of the tryptophan emission spectrum of $0.5 \mu \mathrm{M}$ wild type and K17D/D37K eCBL on urea concentration.................................

Figure 3.1. Agarose (1\%) gel of the 5' and 3' amplicons, labelled R and L, respectively, for

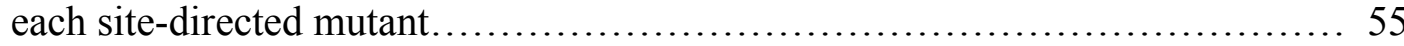

Figure 3.2. Agarose (1\%) gel of the re-assembly products of the second PCR step in the construction of the site-directed mutants via the overlap-extension method........ 55

Figure 3.3. SDS-PAGE gel stained with Comassie Blue of the purified variants eCBL-A207 


\section{Abbreviations}

Assay buffer

50mM Tris buffer, $\mathrm{pH} 8.5,2 \mu \mathrm{M}$ PLP, $2 \mathrm{mM}$ DTNB and 0.004-6.2Mm of L-cystathionine

AVG

Buffer A

CBL

CBS

CGL

CGS

$\mathrm{C}_{\mathrm{m}}$

DNA

DTNB

E. coli

E.C.

eCBL

EDTA

$\mathrm{H}_{2} \mathrm{~S}$

IPTG
Aminoethoxyvinylglycine

$50 \mathrm{mM}$ potassium phosphate, $\mathrm{pH} 8,10 \mathrm{mM}$ imidazole

Cystathionine $\beta$ - lyase

Cystathionine $\beta$ - synthase

Cystathionine $\gamma$ - lyase

Cystathionine $\gamma$ - synthase

The chemical denaturation midpoint

Deoxyribonucleic acid

5, 5' -Dithiobis- (2-nitrobenzoic acid)

Escherichia coli

Enzyme Commission

Escherichia coli cystathionine $\beta$ - lyase

Ethylenediaminetetraacctic acid

Hydrogen sulfide

Isopropyl- $\beta-D$ - thiogalactopyranoside 


\begin{tabular}{|c|c|}
\hline$\lambda_{\mathrm{ex}}$ & Excitation wavelength \\
\hline L-Cth & L- Cystathionine \\
\hline L-Cys & L- Cysteine \\
\hline L-Hcys & L- Homocysteine \\
\hline $\mathrm{NaCl}$ & Sodium Chloride \\
\hline $\mathbf{N H}_{3}$ & Ammonia \\
\hline Ni-NTA & $\mathrm{Ni}$ - nitrile triacetic acid \\
\hline $\mathrm{NO}_{2}$ & Nitrogen dioxide \\
\hline OD & Optical Density \\
\hline OE-PCR & Overlap- extension polymerase chain reaction \\
\hline PCR & Polymerase chain reaction \\
\hline PLP & Pyridoxal 5'- phosphate \\
\hline rpm & revolutions per minute \\
\hline S. cerevisiae & Saccharomyces cerevisiae \\
\hline Storage buffer & $\begin{array}{l}50 \mathrm{mM} \text { potassium phosphate, } \mathrm{pH} 7.8 \text {, containing } 1 \mathrm{mM} \text { EDTA, } 1 \mathrm{mM} \\
\text { dithiotrietol and } 20 \mu \mathrm{M} \text { PLP }\end{array}$ \\
\hline SAM & $S$-adenosyl- methionine \\
\hline SDS-PAGE & Sodium dodecyl sulfate polyacrylamide gel electrophoresis \\
\hline TNB & 3-thio-6-nitrobenzoate \\
\hline
\end{tabular}


Tris

UV

yCGL
Tris-[hydroxymethyl] aminomethane

Ultraviolet

Yeast Cystathionine $\gamma$ - lyas 


\section{Introduction}

\subsection{Importance of Protein Engineering}

Technologies that enable the use of renewable energy sources or reduction in energy consumption are becoming highly sought after to address environmental issues, such as global warming (Ordu et al., 2012; Keleş and Bilgen, 2012). This has given rise to the investigation of methods to reduce industry dependence on fossil fuels, and one way of accomplishing this is to increase the energy efficiency of manufacturing and industrial processes. Biocatalysis relies on the use of natural catalysts, such as enzymes, to catalyze chemical reactions. The ability of enzymes to perform highly selective reactions under mild conditions (i.e. those found inside a living cell), provides the opportunity to reduce both energy requirements (e.g. by reducing the need to heat reactions to the high temperatures typical of synthetic organic chemistry) and environmental impact (e.g. by eliminating the need for toxic metals as catalysts and organic solvents), while concomitantly increasing the yields of processes that require chemical synthesis or transformations (Ordu et al., 2012). Examples of industrial applications of enzymes include agri-food, pharmaceutical, pulp and paper, cosmetics, sanitation, textiles and many others. However, naturally occurring enzymes are adapted to the environment in which they evolved and so are poorly suited for the non-physiological conditions (e.g. extremes of temperature or pH, presence of organic solvents) typical of industrial processes (Lehmann and Wyss, 2001). A further challenge in the use of enzymes is their evolutionary limitation to reactions required by living systems. Protein engineering offers the opportunity to modify enzymes in order to optimize their stability and/or substrate and reaction specificity and opens the door for the development of biocatalysts for a range of biotechnological and industrial applications (Carter, 2011). 
An important feature of enzymes is their exquisite control of reaction specificity. This ability is particularly important for enzymes that employ catalytically versatile cofactors, such as pyridoxal 5' phosphate (PLP). The PLP cofactor catalyzes a range of transformations of amino acid substrates, including transamination, racemization, decarboxylation and side-chain rearrangements. The cofactor provides the catalytic power, while substrate and reaction specificity is regulated by the protein component of the enzyme (Eliot and Kirsh, 2004; Jasonius, 1998). The versatility of PLP and diversity of the reactions catalyzed are demonstrated by the more than 140 distinct Enzyme Commission (E.C.) numbers assigned to enzymes reliant on this cofactor and their presence in five of the six enzyme classes (EC 2 - transferases, EC 3 hydrolases, EC 4 - lyases, EC 5 - isomerases and EC 6 - ligases) defined by the Enzyme Nomenclature Committee of the International Union of Biochemistry and Molecular Biology (Percudani and Peracchi, 2003; Christen, 2001, Eliot and Kirsch, 2004). This demonstrated catalytic versatility provides the opportunity to engineer PLP-dependent enzymes that catalyze novel transformations of amino acid substrates for biotechnological applications (Percudani and Peracchi, 2003; Woycechowsky and Hilvert, 2006). Techniques for engineering enzymes to modify or improve specificity or catalytic efficiency have increased considerably over the past 10-15 years. While directed evolution methods allows selection following rounds of random mutagenesis, particularly when modifying stability, a solid foundation in the structure-function relationships of an enzyme is acknowledged as a requisite basis for successful engineering of substrate and/or reaction specificity (Agarwal et al., 2012; Hult, 2007).

The PLP-dependent enzymes of the transsulfuration and reverse transsulfuration pathways provide a useful investigation model in PLP dependent enzymes for substrate and reaction 
specificity because they effectively catalyze various side-chain reactions of similar amino acid substrates (Lodha and Aitken, 2011). The second enzyme of each of these pathways catalyzes a distinct hydrolysis reaction of the common intermediate cystathionine to yield either cysteine (cystathionine $\gamma$-lyase) or homocysteine (cystathionine $\beta$-lyase). Cystathionine $\beta$-lyase (CBL) and cystathionine $\gamma$-lyase (CGL) are known to catalyse cystathionine (CTT) in the transsulfuration and reverse transsulfuration pathways to produce cysteine and homocysteine respectively. A greater understanding of the structure-function relationships and the factors underlying specificity in these enzymes will provide insights useful for the design of novel activities and can give rise to new information that can help with the formation of antimicrobials as well as therapeutics for hypertension (Steegborn et al., 1999).

\subsection{PLP-dependent Enzymes}

Pyridoxal 5' phosphate is derived from vitamin B6 and is found in all organisms (Toney, 2004). This catalytically versatile cofactor is able to conduct transformations of amino acid substrates, including transamination, racemization, decarboxylation and side-chain rearrangements (Figure 1.1) (Percudani and Peracchi, 2003; Jansonius, 1998). The interactions between active site residues and the aromatic portion of the cofactor modify the electron sink character of the PLP co-factor, thereby contributing to reaction specificity (Hayshi et al., 1990). Other factors regulating specificity continue to be explored. In the resting, internal aldimine state of a PLPdependent enzyme, the cofactor is covalently bound via a Schiff-base linkage to the $\varepsilon$-amino group of the active site lysine residue. The latter acts as the catalytic base when it is replaced, via transaldimination, by the $\alpha$-amino group of the amino acid substrate, thereby forming the external aldimine. 


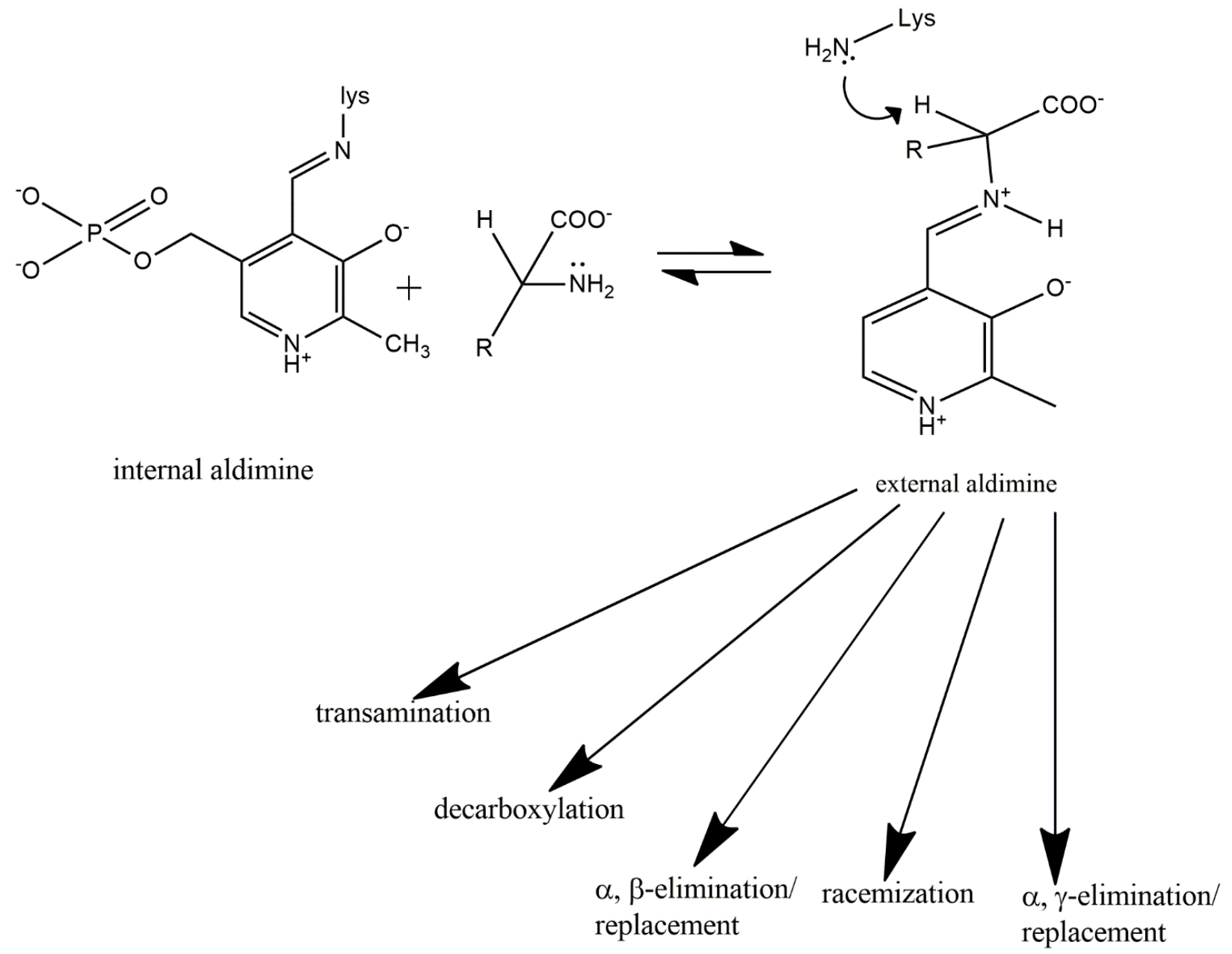

Figure 1.1. The transaldimination reaction, in which the $\alpha$-amino group of the substrate replaces the $\varepsilon$-amino group of the active-site lysine in Schiff base linkage with the cofactor is the first step in a range of PLP-catalyzed transformations of amino acid substrates (Adapted from Toney, 2004; Aitken and Kirsh, 2005). 
Cleavage of one of the three $\mathrm{C} \alpha$ bonds, other than the amino group, then starts the process of the specific substrate transformation catalyzed by the given PLP-dependent enzyme (Schneider et al., 2000).

Enzymes dependent on the PLP cofactor are classified into five distinct structural, or fold, groups. The most common and best classified of these is fold type I (Percudani and Peracchi, 2003; Schneider et al., 2000). Aminotransferases and decarboxylases, as well as the enzymes catalyzing $\beta$ and $\gamma$ elimination and replacement reactions which comprise the $\gamma$-subfamily, share a common overall and active site structure and are classified as members of this fold type. The $\gamma$ subfamily of fold-type I includes three of the four enzymes of the transsulfuration pathways: cystathionine $\gamma$ - synthase (CGS), cystathionine $\beta$ - lyase (CBL) and cystathionine $\gamma$ - lyase (CGL) (Percudani and Peracchi, 2003). These homotetrameric enzymes are organized as a dimer of functional dimers in which residues of each monomer pair contribute to the active site of the two active sites in each dimer (Schneider et al., 2000). The fourth enzyme of the transsulfuration pathways, cystathionine $\beta$-synthase (CBS), typical of fold type II enzymes, catalyzes a $\beta$ replacement reaction and possesses a regulatory domain that is distinct from the catalytic core. The smaller fold-type III and IV families comprise the D-amino acid aminotransferases and the alanine racemases, respectively. Fold- type $\mathrm{V}$ is unique as the glycogen phosphorylases of this group rely on the phosphate group of the cofactor for proton transfer rather than the aromatic ring (Percudani and Peracchi, 2003; Schneider et al., 2000)

\subsection{The Transsulfuration and Reverse Transsulfuration Pathways}

One of the central biological functions of amino acids is their role as the building blocks of proteins (Brosnan and Brosnan, 2006). The two sulfur-containing proteinogenic amino acids, 
methionine and cysteine, are also the precursors of $S$-adenosylmethionine (SAM) and glutathione, compounds of primary importance in cellular one-carbon metabolism and redox chemistry, respectively. They are metabolically linked through the transsulfuration pathways, which interconvert cysteine and homocysteine, the immediate precursor of methionine (Figure 1.2) (Stipanuk, 2004; Lodha and Aitken, 2011). Bacteria and plants synthesize cysteine de novo and via the action of the enzymes of the transsulfuration pathway, CGS and CBL, replace the activating group of $O$-succinyl (bacteria) or $O$-phospho-homoserine (plants) with the sulfhydryl moiety of cysteine to form homocysteine (Brosnan and Brosnan, 2006; Stipanuk, 2004). These enzymes have been identified as targets for the development of anti-microbial compounds and herbicides, as they are unique to bacteria and plants (Steegborn et al., 1999; Aitken and Kirsh, 2005; Clausen, 1998). In contrast, methionine is an essential amino acid for mammals as it must be obtained through diet. The trans-methylation pathway converts methionine to homocysteine, via SAM and $S$-adenosylhomocysteine. Homocysteine can be either re-methylated to regenerate methionine or shunted to cysteine, via the reverse transsulfuration pathway, common to animals and fungi (Aitken and Kirsh, 2005). 


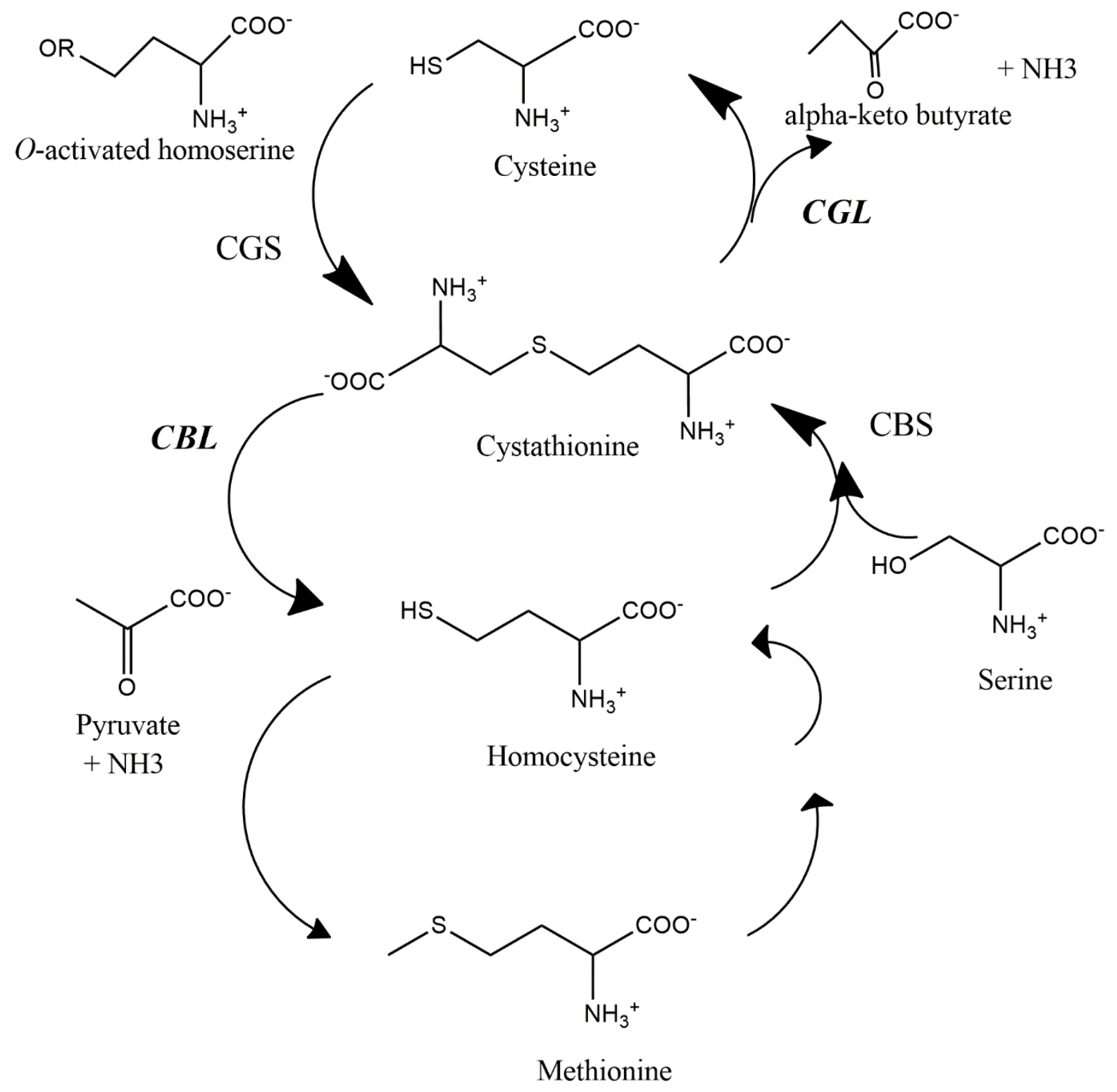

Figure 1.2. The bacterial/plant transsulfuration (CGS and CBL) and mammalian/yeast reverse transsulfuration (CBS and CGL) pathways which interconvert cysteine and homocysteine, the immediate precursor of methionine. Cystathionine is the common intermediate of both pathways. (Adapted from Steegborn et al., 1999). 


\subsection{Comparison of CGL and CBL}

Cystathionine $\beta$-lyase (CBL) and cystathionine $\gamma$-lyase (CGL) are the second enzymes in the transsulfuration and reverse transsulfuration pathways, respectively (Figure 1.2). These two enzymes are members of the $\gamma$-subfamily of fold type I and, while they share only $30 \%$ amino acid sequence identity, adopt a common tertiary and quaternary structure asboth homotetrameric enzymes are organised as a dimer of catalytic dimers, (Lodha and Aitken, 2011; Farsi et al., 2009). Both CBL and CGL catalyze the hydrolysis of cystathionine but cleave the substrate at the $\beta$ and $\gamma$-positions, producing homocysteine and cysteine, respectively (Lodha and Aitken, 2011; Jansonius, 1998; Dorbic et al., 2000; Aitken et al., 2011). The distinct reaction specificity of these enzymes, which share a common structure and catalyze the cleavage of the same pseudo-symmetric amino acid substrate, provides a useful model system for investigation of the subtle determinants of reaction specificity in PLP- dependent enzymes.An understanding of the structure-function relationships that underlie the distinct reaction specificity of these enzymes will contribute to the foundational understanding required for future studies aiming to engineer these enzymes for use in industrial processes. The methionine biosynthetic pathway has been identified as an interesting target for antibiotic design as it plays an important role in protein DNA metabolism and one-carbon chemistry, in addition to being absent in mammals (Brown and Wright, 2005; Ejim et al., 2007). Enzymes involved in the metabolism of sulphur-containing amino acids are also known to play a role in production of flavour compounds in fermented foods such as cheeses, wines and sausages and, as such, are of interest for biotechnological applications in the agri-food industry (Liu et al., 2008; Ardo, 2006; Yvon and Rijnen, 2001). 


\subsubsection{Protein Architecture}

The Escherichia coli CBL (eCBL) enzyme is encoded by the $M e t C$ gene and each subunit of the homotetramer is 395 amino acids in length (Belfazia et al., 1986; Clausen et al., 1996). Similarly, each of the four identical Saccharomyces cerevisiae CGL (yCGL) subunits is 393 amino acids in length (Messerschmidt et al, 2003). Each eCBL or yCGL monomer is comprised of three structural domains: the N-terminal domain (residues 1-60 and 1-54 of eCBL and yCGL, respectively), the PLP- binding domain (residues 61-256 and 54-252 of eCBL and yCGL, respectively) and the $\mathrm{C}$ - terminal domain (residues 257-395 and 252-393 of eCBL and yCGL, respectively). The $\mathrm{N}$ terminal domain contributes to the active site of the adjacent subunit of a monomer pair and tetramer formation by interacting with the neighboring monomer across the dimer interface. It consists of three $\alpha$-helices, one $\beta$-strand and an extended loop structure. The PLP-binding domain includes most of the residues involved in substrate binding and catalysis. It also consists of an $\alpha / \beta$ structure with a seven stranded $\beta$ sheet at the core, and is connected to the C-terminal domain by an extended $\alpha$-helix. The $\mathrm{C}$ - terminal domain comprises an anti-parallel four-stranded $\beta$-sheet stabilized by packing interactions with three $\alpha$-helices. The active site cleft is situated between the PLP-binding and C-terminal domains (Messerschmidt et al., 2003; Clausen et al., 1996).

The homotetrameric fold-type I enzymes of the transsulfuration pathways associate as a pair of dimers. The association of subunits to form the catalytic dimers includes insertion of the Nterminal domain into the active site of the neighboring monomer, such that each active site is comprised of residues contributed by each subunit of the pair (Clausen et al., 1996;

Messerschmidt et al., 2003). The interactions underlying monomer association are largely conserved between eCBL and yCGL, with the exception of a pair of $~ 25$-residues, situated in the 
amino and carboxy-terminal regions (segments I and II), that adopt distinct conformations (Clausen et al., 1996; Messerschmidt et al., 2003; Manders et al., 2013). In contrast, the association of the homodimers to form the native tetrameric structure is mediated by distinct interactions in eCBL and yCGL. Tetramer formation is mediated by polar and charged residues versus hydrophobic interactions in eCBL and yCGL, respectively. The approximately 20 residues that form the primary dimer interface are distributed through the three subunit domains (Clausen et al., 1996; Messerschmidt et al., 2003). As such, this interface influences active site structure and conformation and may act as a determinant of reaction specificity. To date, there have been no reports probing the dimer interface or exploring the effect of substitutions in this region on the activity or stability of these enzymes.

\subsubsection{Active Site Architecture}

Typical of PLP-dependent enzymes of fold-types I-IV, the PLP cofactor of yCGL and eCBL is covalently bound, via Schiff-base linkage, to the $\varepsilon$-amino group of an active site lysine residue (K210 in eCBL and the corresponding K203 of yCGL) (Clausen et al.,1996; Messerschmidt et al., 2003). The active site is situated in the cleft between the PLP binding and C-terminal domains. Interaction with a conserved aspartate residue (eCBL-D185, yCGL- D178) maintains the positive charge of the N1 nitrogen atom of the pyridine ring, thereby enhancing the electron withdrawing nature of the PLP cofactor. The O3' hydroxyl group forms a hydrogen bond to the side chain of W340 in eCBL, but this residue is replaced by $\mathrm{L} 327$ in yCGL, a difference that may alter the ability of the pyridine ring to stabilize the delocalized carbanion formed upon abstraction of the $\mathrm{C} \alpha$ proton. However, the catalytic efficiency of eCBL is not reduced by substitution of W340 with phenylalanine (Lodha et al., 2010). Therefore, any role that the 
residue at this position plays is subtle and may require communication with other active site residues. The phosphate moiety of the cofactor is anchored by hydrogen bonds to several residues including a conserved tyrosine (eCBL-Y56; yCGL-Y49) and arginine (eCBL-R58;

yCGL-R51). The pyridinium ring of the cofactor engages in $\pi$-stacking interactions with the aromatic ring of a conserved tyrosine (eCBL- Y111; yCGL- Y103) (Clausen et al., 1996; Messerschmidt et al., 2003).

The pseudosymmetric cystathionine substrate of eCBL and yCGL contains the equivalent of two $\mathrm{C} \alpha$ atoms, thereby allowing it to bind in opposite orientations in active sites of these enzymes to enable $\beta$-versus $\gamma$-elimination, respectively (Figure 1.2) (Aitken et al., 2011). The $\alpha$-carboxylate moiety of the substrate forms a salt-bridge with a conserved active site arginine (eCBL-R372, yCGL-R369) and, in the case of eCBL, with the side chain of W340 (yCGL-L327), although the latter plays a secondary role in substrate binding (Aitken et al., 2011; Lodha et al., 2009). The distal carboxylate moiety is fixed by a second salt-bridge to a conserved arginine residue (eCBL$\mathrm{R} 58$, yCG-R51), providing rigidity and precise positioning of the distal portion of the substrate, with respect to the cofactor and catalytic base (Clausen et al., 1996; Messerschmidt et al., 2003; Lodha et al., 2009).

\subsubsection{Reaction Mechanism}

The eCBL enzyme catalyzes the $\beta$-elimination of cystathionine, producing homocysteine, pyruvate and ammonia while the $\gamma$-elimination of yCGL yields cysteine, $\alpha$-ketobutyrate and ammonia (Aiken et al, 2011). This difference requires that the pseudosymmetric cystathionine substrate docks in opposite orientations in the active site of these two enzymes. Although the 
orientation in which the cystathionine is bound to the active site is different for the two enzymes, the initial step of the reaction mechanisms are the same and include transaldimination to form the external aldimine of the cystathionine substrate, followed by abstraction of the $\mathrm{C} \alpha$ proton and stabilization of the resulting carbanion by delocalization in the pyridinium ring of the cofactor (Clausen et al., 1998). The facile $\beta$-elimination of eCBL proceeds with elimination of the homocysteine leaving group and a second transaldmination step to return the enzyme to the resting internal aldimine state. In contrast, proton transfer between $C \beta$ of the substrate and the C4' position of the cofactor is required in the multi-step $\gamma$-elimination catalyzed by yCGL. In both enzymes the catalytic base responsible for proton abstraction and transfer steps is the active site lysine residue (eCBL-K210 and yCGL-K203). Following its release from Schiff base linkage with the cofactor, upon formation of the external aldimine substrate-cofactor complex, movement of the catalytic base is proposed to be guided by a pair of residues conserved among the enzymes of the $\gamma$-subfamily (Y56 and S339 of eCBL and Y49 and S334 of yCGL) (Lodha and Aitken, 2011; Jaworski et al., 2012). These residues tether the lysine (eCBL-K203) and orient it in a position that restricts access of the $\varepsilon$-amino group to $\mathrm{C} \alpha$ of the substrate-cofactor complex in eCBL. In contrast, the corresponding K203 in yCGL must swing between $\mathrm{C} \alpha$ and $\mathrm{C} \beta$ of the substrate and the C4' position of the cofactor (Messerschmidt et al., 2003; Aitken et al., 2011). Although yCGL-K203 is proposed to be tethered by the same conserved serine (yCGLS334, eCBL-S339) and tyrosine (yCGL-Y49, eCBL-Y56), comparison of the structures of yCGL and eCBL shows a third nearby residue, not conserved between these enzymes (yCGL-S200; eCBL-A207). 


\section{Cystathionine $\beta$ - lyase mechanism}

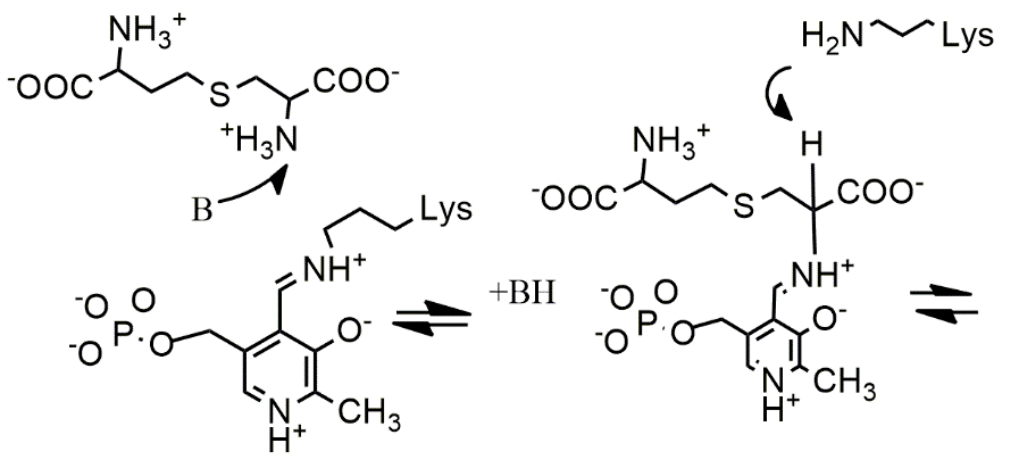

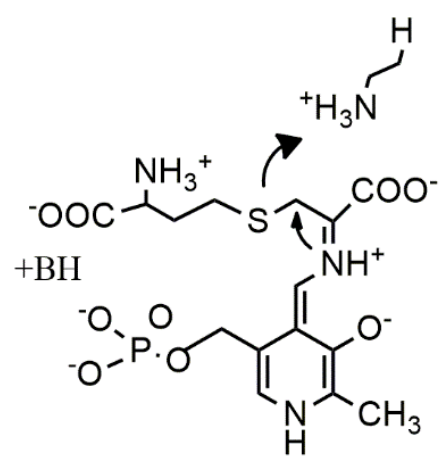

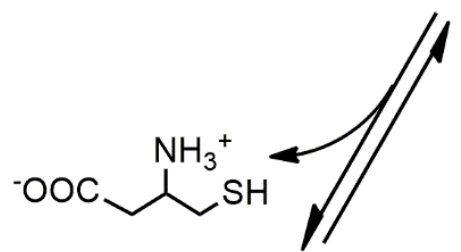

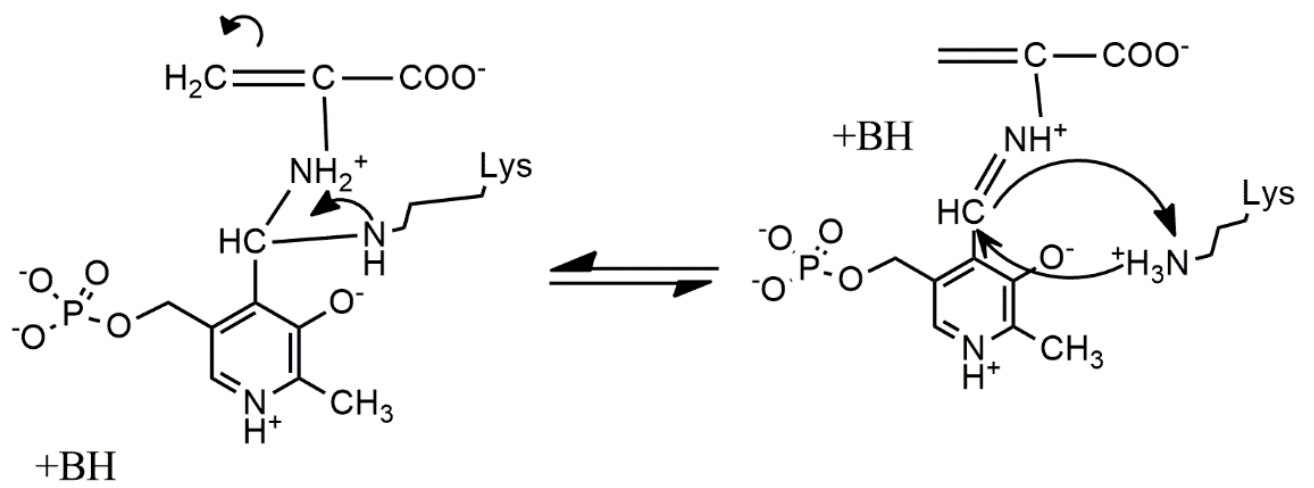

$+\mathrm{BH}$<smiles>C=C=C=C</smiles>

Figure 1.3. The chemical mechanism of the $\gamma$-elimination of cystathionine catalyzed by CBL, to the point of the release of the cysteine product (Adapted from Messerschmidt et al., 2003; Clausen et al., 1996). 


\section{Cystathionine $\gamma$ - lyase mechanism}

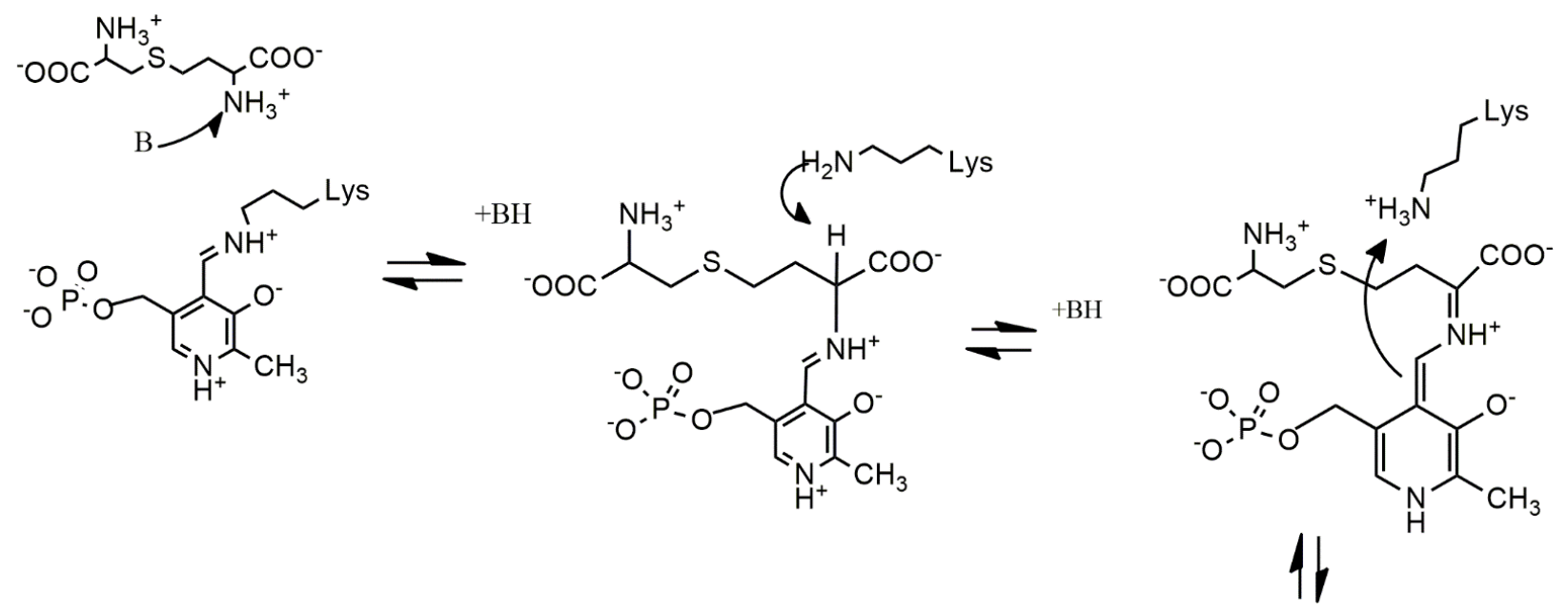

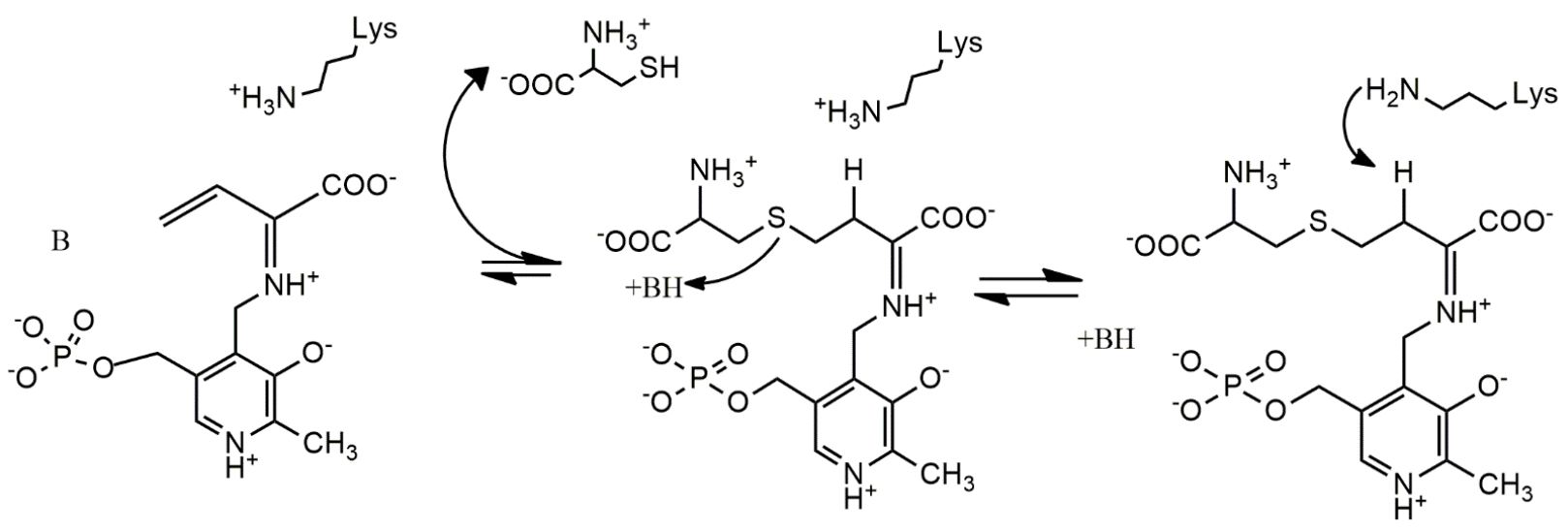

Figure 1.4. The chemical mechanism of the $\gamma$-elimination of cystathionine catalyzed by CGL, to the point of release of the homocysteine product (Adapted from Messerschmidt et al., 2003). 
In the context of yCGL, residue S200 may act to modulate the movements permitted by the catalytic base via formation of a hydrogen bond to the side chain of K203 that cannot be formed by the corresponding $\mathrm{A} 207$ of eCBL as the side chain of this residue lacks the ability to form hydrogen bonds (Clausen et al., 1996; Messerschmidt et al., 2003).

\subsection{Objectives}

Previous studies by our lab have characterized the roles for many of the active site residues of eCBL, eCGS and yCGL. These studies identified the conserved arginine residues that bind the carboxylate groups of the substrate(s), probing the role of residues interacting with the substrate(s) and cofactor. They also demonstrated the role of the conserved tyrosine (eCGS-Y46, eCBL-Y56, yCGL-Y49) and serine (eCGS-S326, eCBL-S339, yCGL-S334) residues in tethering the catalytic base (Farsi et al., 2009; Lodha et al., 2010; Lodha and Aitken, 2011; Aitken et al., 2011; Jaworski et al., 2012; Manders et al., 2014). The distinct $\beta$ versus $\gamma$-elimination reaction specificity of the structurally conserved eCBL and yCGL enzymes provides an ideal system to probe the subtle factors that regulate activity and specificity. Studies exploring these factors will enable the continued development of our understanding of the mechanisms whereby PLPdependent enzymes regulate substrate and reaction specific. This will allow for building a framework that will facilitate future studies aiming to engineer these enzymes to harness the power of this catalytically versatile cofactor for use in various industrial processes. .

The objectives of the research described in this thesis are:

1. The preliminary exploration of the contribution of a pair of salt-bridges (K5-D382 and K17D37), spanning the dimer interface of eCBL, to enzyme activity and stability. To achieve this 
goal, the effect of charge-reversal substitutions of the four residues involved, on the catalytic efficiency, stability and fluorescence-monitored urea denaturation of eCBL, was investigated.

2. A direct role for yCGL-S200 and an indirect role for S50, mediated by the adjacent Y49, in influencing the positioning of the $\varepsilon$-amino group of the active site lysine, in the context of yCGL, were postulated. In order to test this hypothesis, a series of substitutions (S50A, S50G and S200A of yCGL and G57S and A207S of eCBL) probing the role of the side-chain hydroxyl moiety of the serine residues of yCGL, in the context of both enzymes, was characterized. 
Chapter 2: Investigation of a pair of charged interactions at the dimer interface of cystathionine $\beta$-lyase 


\subsection{Abstract}

Cystathionine $\beta$-lyase, the second enzyme of the transsulfuration pathway, catalyzes the $\beta$ elimination of L-cystathionine to produce L-homocysteine, ammonia and pyruvate. The dimer interface of the homotetrameric Escherichia coli $\mathrm{CBL}$ (eCBL) enzyme is characterized by charged and polar residues that form hydrogen-bonding and salt-bridge interactions in comparison to the Saccharomyces cerevisiae (yCGL) enzyme whose dimer interface includes hydrophobic interactions. The dimer interface, in both eCBL and yCGL, is close to the four active sites of the homotetramer, and the N-terminal domain of each monomer contributes both to the active site of the adjacent monomer in each catalytic dimer and to the dimer interface. As such the dimer interface interactions that distinguish eCBL and yCGL may play a role as determinants of reaction specificity.

The K5/D382 and K17/D37 salt bridges connect residues from the two catalytic dimers and are located at the exterior edge of the dimer interface. A series of four single and two double chargereversal, site-directed variants of these residues were constructed and their effect on the fluorescence-monitored urea denaturation profile and the kinetic parameters of eCBL were investigated. The role of these salt-bridge interactions in the proper folding and stability of this enzyme is demonstrated by our inability to express and purify the K17D and D382K variants as well as the $0.5-1 \mathrm{M}$ decrease in the primary transitions of the denaturation profile of the $\mathrm{K} 5 \mathrm{D}$ and D37K single-substitution variants, which were all expected to destabilize dimer association by co-locating pairs of identical charges. As anticipated, the shifts in the denaturation profiles of the double-substitution variants, which maintain but invert the salt bridges, are relatively minor. However, even these subtle changes are conveyed to the active site as observed in the 11-21-fold reductions in the catalytic efficiency and the early release from quenching of W188 in the urea 
denaturation profiles of the four purified variants, which are likely due to changes in the architecture and/or dynamics of the active site. 


\subsection{Introduction}

The efficiency and exquisite substrate and reaction specificity of enzymes makes them attractive tools for efforts to increase productivity while reducing the energy requirements and environmental cost of manufacturing or industrial processes involving chemical transformations (Ordu et al., 2012). However, the subset of reactions for which naturally occurring biocatalysts can be found is much smaller than the range of possible chemical transformations, such that enzymes do not currently exist for many industrial applications. The variety of enzymes available provides the opportunity to select one to use as a scaffold, or starting point, for modification of the targeted properties (Lehmann and Wyss, 2001). Selection of a specific enzyme as the starting point is based on criteria that include similarity of reaction type and/or substrate specificity of a naturally occurring enzyme and the desired endpoint. Although developments in molecular biology and protein engineering techniques have evolved to the point that enzyme properties, including stability and specificity, can be modified given the complexity of the structure-function relationships of these very large macromolecules, each case is a unique and distinctly challenging undertaking. The vastness of sequence space limits the potential of directed evolution methods, based on the random introduction of point mutations, particularly for modification of specificity. As such, the success of protein engineering studies is enhanced by detailed biochemical characterization and structure-function analysis of the enzyme selected as the starting point for modification of substrate and/or reaction specificity (Carter, 2011).

Enzymes reliant on cofactors, such as PLP, provide a wide scope of opportunities as starting points for protein engineering, as well as for studies exploring the structure-function relationships that underlie specificity, as the catalytic versatility of the cofactor allows many possible transformations of amino acid substrates (e.g. decarboxylation, side-chain 
rearrangement, deamination, racemisation, transamination) and is controlled by the protein component of the enzyme. The PLP-dependent enzymes of the transsulfuration and reverse transsulfuration pathways provide a particularly useful model for exploring the structure-function relationships underlying substrate and reaction specificity because, as members of the $\gamma$ subfamily of fold-type I of PLP enzymes, they share a common overall structure and several conserved active site residues, but catalyze distinct transformations of similar or identical substrates (Lodha and Aitken, 2011). Therefore, the development of a greater understanding of the structure-function relationships and the factors underlying specificity in these enzymes will provide insights useful for the design of novel activities. The enzymes of the bacterial transsulfuration pathway are also of interest as targets for the design of novel antimicrobial compounds (Steegborn et al., 1999).

Cystathionine $\beta$ - lyase (CBL) catalyzes the hydrolysis of L-cystathionine in the second step of the bacterial and plant transsulfuration pathway, which converts L-cysteine to L-homocysteine, the immediate precursor of L-methionine (Ejim et al., 2007; Clausen et al., 1996). It is a member of the $\gamma$-subfamily of fold-type I of PLP-dependent enzymes and closely related in structure to cystathionine $\gamma$-synthase, which precedes it in the transsulfuration pathway, and cystathionine $\gamma$ lyase, the second enzyme of the reverse transsulfuration pathway of mammals and yeast (Figure 2.1) (Aitken et al., 2011). These enzymes adopt a homotetrameric structure comprised of a pair of catalytic dimers, in which each monomer contributes to both active sites of the dimer (Clausen et al., 1996, Ejim et al., 2007, Laber et al., 1995). The dimer interface of Escherichia coli CBL (eCBL) is characterized by polar and charged residues that form a series of hydrogen bonds and salt-bridges (Clausen et al., 1996). 


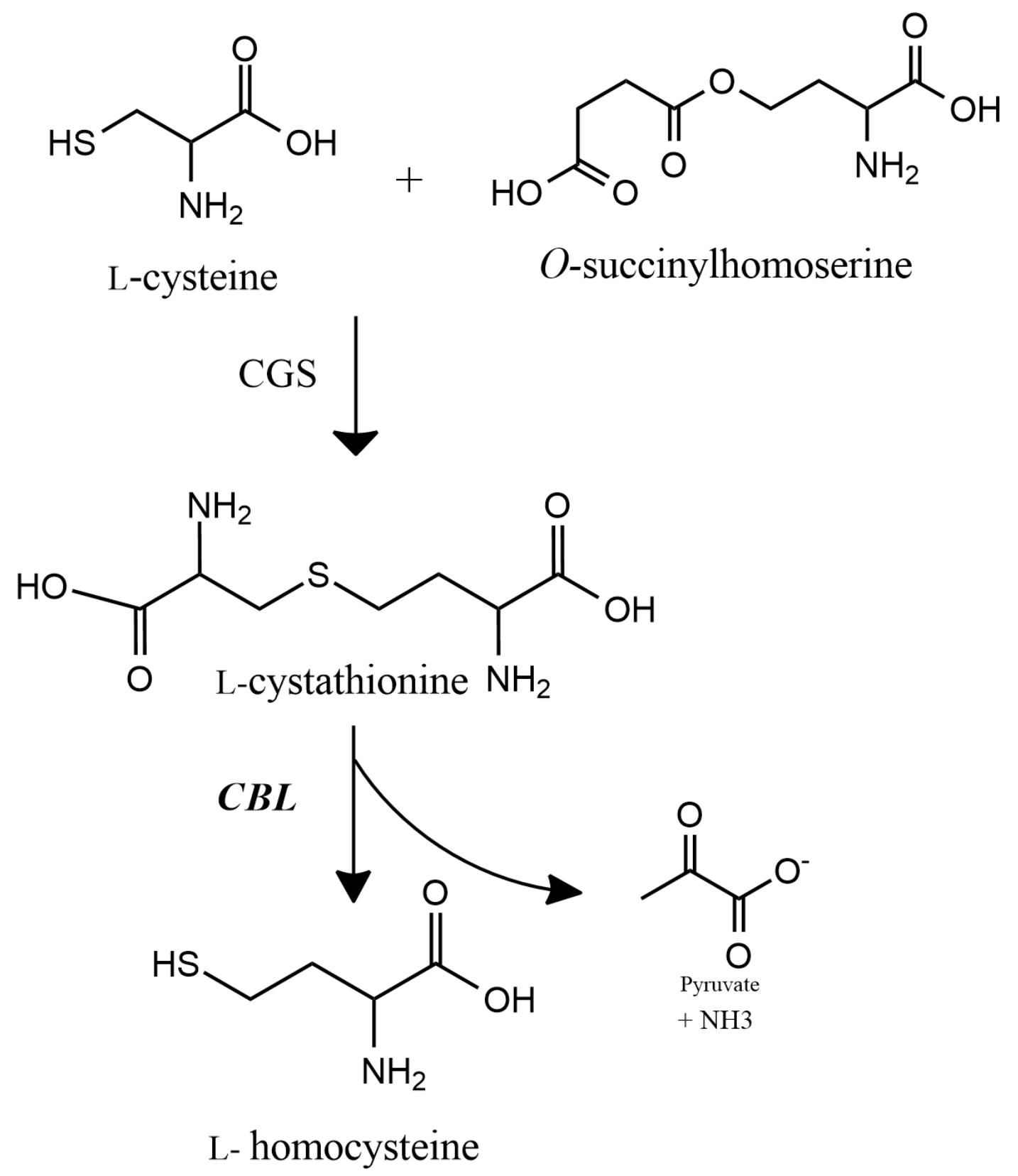

Figure 2.1: The transulfuration pathway of bacteria showing the reactions catalyzed by cystathionine $\gamma$-synthase and cystathionine $\beta$-lyase (adapted from Manders et al., 2013). 
In contrast, the catalytic dimers of $S$. cerevisiae CGL (yCGL) associate primarily via nonpolar, hydrophobic interactions, a difference which may contribute to the specificity of these two enzymes as the active sites are situated proximal to the dimer interface (Messerschmidt et al., 2003).

Ongoing work in our lab (by Duale Ahmed, $\mathrm{PhD}$ candidate and Edgar Abouassaf, PhD 2015), is investigating the charged residues R30, D217, D247, Y250 and R254 which are located at the centre of the eCBL dimer interface. The preliminary results of charge-inversion substitutions of these residues, individually (e.g R30D, D247R single substitutions) and in salt-bridge pairs (e.g. R30D/D247R double substitution), show a reduction in catalytic efficiency of up to five orders of magnitude (unpublished results), suggesting that these residues play an important role in maintaining and stabilizing the quaternary structure of eCBL and that interactions and/or conformational changes at the dimer interface have a direct impact on enzyme activity.

The interactions at the periphery of the dimer interface have not yet been probed. These include the salt bridge between residues $\mathrm{K} 5$ and $\mathrm{D} 382$, of the $\mathrm{N}$ - and $\mathrm{C}$-terminal domains, and $\mathrm{K} 17$ and D37, both of the N-terminal domain (Clausen et al., 1996). Interestingly, the K5-D382 salt bridge is conserved in yCGL, while the K17-D37 interaction is replaced by a pair of hydrophobic residues (Messerschmidt et al., 2003). As an initial step in characterization of the role of a charged (eCBL) versus hydrophobic (yCGL) interface as a determinant of reaction specificity, the effect of charge-reversal substitutions, targeting these two interactions situated at the periphery of the dimer interface, are explored in this study. A series of site-directed variants was constructed to investigate the effect of single (e.g. K5D) and paired (e.g. K5D/D382K) charge reversal mutations on the catalytic efficiency, stability and fluorescence-monitored urea denaturation of eCBL. 


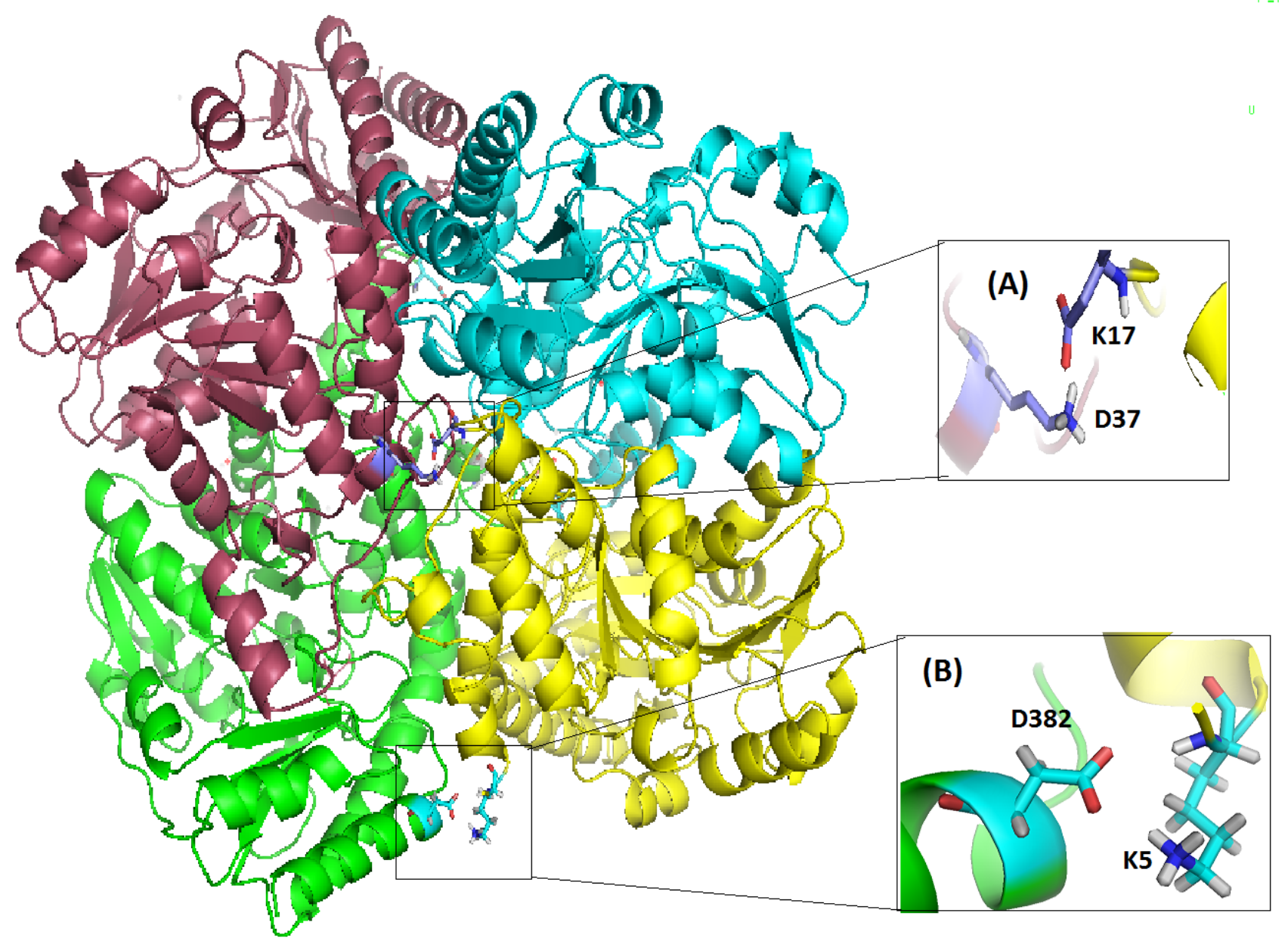

Figure 2.2: A cartoon representation of the eCBL tetramer, distinguishing each subunit by colour. Situated at the periphery of the dimer interface are two pairs of charged residues which form a pair of salt bridge interactions that participate in eCBL tetramer formation: (A) K17-D37 and (B) K5-D382 (PDB: 1CL1) (Clausen et al., 1996). 
2.3 Materials and Methods

\subsubsection{Reagents}

Ampicillin, 5, 5'-dithio-bis-(2-nitrobenzoic acid) (DTNB) and imidazole were purchased from Fisher Scientific and L-cystathionine was from Sigma. Restriction endonucleases, Antarctic phosphatase and T4 DNA ligase were obtained from New England Biolabs. Nickelnitrilotriacetic acid (Ni-NTA) resin was from Qiagen. Oligonucleotide primers were synthesized by Integrated DNA Technologies.

\subsubsection{Construction of eCBL Site-Directed Mutants}

Overlap-extension polymerase chain reaction (OE-PCR) with the pSECseq0 (GGC GTC AGG CAG CCA TCG GAA GCT G) and pSECseq7r (GCC CGC CAC CCT CCG GGC CGT TGC TTC GC) primers, which flank the multiple cloning site of the vector, and the respective mutagenic primers was employed to construct the K5D, K17D, D37K, D382K, K5D/D382K and K17D/D37K charge-reversal substitution variants of eCBL (Table 2.1). The resulting amplicons were gel purified prior to insertion between the NdeI and BamHI sites of the pTrc3 plasmid, which encodes an N-terminal 6x-His affinity tag that enables purification of the recombinant protein using immobilized metal ion affinity chromatography. The restriction-digested pTrc3 vector was treated with Antarctic phosphatase prior to ligation to reduce background due to reannealing of the digested vector. The pTrc3-eCBL constructs were transformed into $E$. coli ER1821 metC::cat strain in which the eCBL coding sequence is replaced by that of chloramphenicol acetyltransferase, thereby precluding contamination with the wild-type E. coli enzyme (Farsi et al., 2009). 
Table 2.1: Sequences of the primers employed to construct the site-directed mutants. ${ }^{a}$

\begin{tabular}{|l|l|}
\hline eCBL & Primer Sequence \\
variants & \\
\hline K5D & 5'-ggcggacaaagatcttgatactcaactgg \\
\hline K17D & 5'-ggacgcagcgataaatacactctcg \\
\hline D37K & 5'-gctggtctttaaaagtgtagaagc \\
\hline D382K & 5'-ggaagatgtcgacaaactgattgccg \\
\hline
\end{tabular}

${ }^{a}$ All mutagenic primers were obtained from Duale Ahmed, $\mathrm{PhD}$ candidate (Aitken lab). The second mutagenic primer is the reverse compliment of the primers listed above 
The full coding regions and plasmid junctions of each site-directed mutant were sequenced by BioBasic prior to expression and purification, to ensure only the desired mutation(s) were present.

\subsubsection{Expression and Purification of the Site-Directed Variants}

A 100-mL overnight culture of ER1821 metC::cat cells containing the given $\mathrm{p}$ Trc3-eCBL construct in rich growth media $(50 \mathrm{mM}$ potassium phosphate containing Vogel Bonner salts and both $7.2 \mathrm{~g}$ tryptone and $14.4 \mathrm{~g}$ yeast extract per litre) and $50 \mu \mathrm{g} / \mathrm{mL}$ each of ampicillin and chloramphenicol was prepared (Vogel and Bonner, 1956). Three Fernbach flasks, containing 1L of rich media ( $3 \times 1 \mathrm{~L}$ ), were each inoculated with $20 \mathrm{ml}$ of overnight culture and incubated at 37 ${ }^{\circ} \mathrm{C}$ and $200 \mathrm{rpm}$ until the $\mathrm{OD}_{600}$ reached 2.5. Expression was induced with $0.15 \mathrm{mM}$ IPTG, followed by incubation for a further 18 hours. The cells were harvested by centrifugation at 5000 rpm for $10 \mathrm{~min}$ and the pellets were washed in $0.85 \% \mathrm{NaCL}$, centrifuged at $5000 \mathrm{rpm}$ for $15 \mathrm{~min}$ and stored at $-80^{\circ} \mathrm{C}$.

The cell pellets were re-suspended in buffer A (50mM potassium phosphate, $\mathrm{pH} 7.8,20 \mu \mathrm{M}$ PLP and $10 \mathrm{mM}$ imidazole), containing $1 \mathrm{mg} / \mathrm{mL}$ of lysozyme, and incubated on ice for $20 \mathrm{mins}$. The cell lysate was sonicated ( 8 cycles of 30 seconds at $50 \%$ duty cycle) and centrifuged at 15000 rpm at $4{ }^{\circ} \mathrm{C}$ for 60 minutes. The resulting supernatant was loaded onto a $1.0 \times 10 \mathrm{~cm}$ column of nickel- nitrilotriacetic acid (Ni-NTA) resin, equilibrated with 20 column volumes of buffer A and the affinity-tagged protein was eluted with a $200-\mathrm{mL}$ linear gradient of $10-200 \mathrm{mM}$ imidazole in buffer A. The fractions collected were assessed for enzyme purity via SDS-PAGE and those containing no visible contaminants were pooled, concentrated and dialyzed against storage buffer (50mM potassium phosphate, $\mathrm{pH} 7.8$, containing $1 \mathrm{mM}$ EDTA, $1 \mathrm{mM}$ dithiotrietol and $20 \mu \mathrm{M}$ 
PLP). Glyercol was added to $20 \% v / v$ before storage at $-80^{\circ} \mathrm{C}$. The protein concentration of each eCBL construct was determined spectroscopically $\left(\varepsilon_{280}=51,105 \mathrm{M}^{-1} \mathrm{~cm}^{-1}\right)$ (Clausen et al. 1997).

\subsubsection{Determination of Steady State Kinetic Parameters for the Hydrolysis of L-cystathionine}

Kinetic parameters of the site-directed variants were assessed in the context of their ability to complement E.coli strains deficient in eCBL activity and to alter the reaction specificity of the enzyme in comparison to the wild-type eCBL (Farsi et al., 2009). Enzyme activity was measured in a total reaction volume of $100 \mu \mathrm{L}$ at $25^{\circ} \mathrm{C}$ with a spectramax 390 spectrophometer (Molecular devices). The assay buffer consisted of 50mM Tris buffer, $\mathrm{pH}$ 8.5, $2 \mu \mathrm{M}$ PLP, 2mM DTNB and 0.004-6.2Mm of L-cystathionine. Pyridoxal 5'-phosphate is included in the buffer to ensure cofactor saturation of the enzymes assayed. The hydrolysis of L-cystathionine was detected ( $\varepsilon_{412}$ $=13,600 \mathrm{M}^{-1} \mathrm{~s}^{-1}$ ) via the reaction of the free thiol of the L-homocysteine product with $5,5^{\prime}$ dithiobis- (2-nitrobenzoic acid) (DTNB) (Figure 2.3) (Farsi et al., 2009; Ellman, 1959). A background reading was recorded before initiation of the reaction by the addition of eCBL (0.675-2.17 $\mu \mathrm{M}$, depending on the activity of the particular site-directed variant) for all assays. The resulting data was fit to the Michaelis-Menten equation (equation 2.1) to determine $\mathrm{k}_{\mathrm{cat}}$ and $\mathrm{K}_{\mathrm{m}}$ and the catalytic efficiency $\left(\mathrm{k}_{\mathrm{cat}} / \mathrm{K}_{\mathrm{m}}\right)$ was obtained independently from equation 2.2. Data were fit by nonlinear regression with the SAS software package (SAS Institute, Cary, NC).

$$
\frac{v}{[E]}=\frac{k_{c a t} \times[S]}{K_{m}+[S]}
$$

$$
\frac{v}{[E]}=\frac{k_{c a t} / K_{m} \times[S]}{{ }_{1+}[S] / K_{m}}
$$




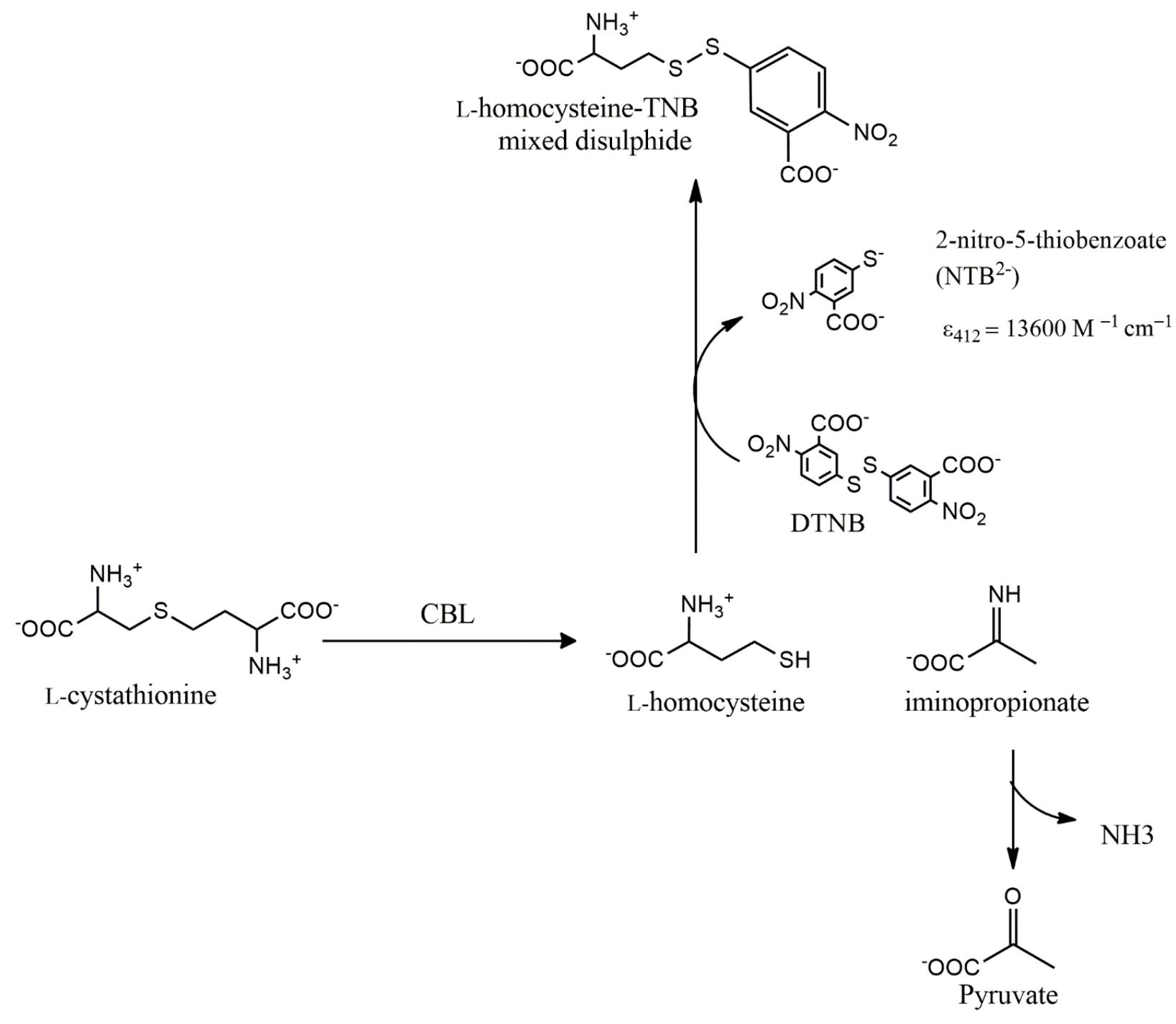

Figure 2.3: The DTNB assay used to assay the L-cystathionine hydrolysis activity of eCBL (Lodha and Aitken, 2011). 


\subsubsection{Stability Studies of wt-eCBL and the Respective Site-Directed eCBL Variants}

The unfolding of the wild-type and variant eCBL enzymes was examined via fluorescencemonitored, urea denaturation. The enzyme was diluted to a final concentration of $0.5 \mu \mathrm{M}$ in 20 $\mathrm{mM}$ phosphate buffer $(\mathrm{pH} 7.5)$ containing $0-7.5 \mathrm{M}$ urea and incubated for 16 hours at room temperature. Fluorescence spectra were recorded $\left(\lambda_{\mathrm{ex}}=295 \mathrm{~nm}\right)$ between $300-380 \mathrm{~nm}$ at $25^{\circ} \mathrm{C}$ with a Cary Eclipse (Varian) spectrofluorometer (excitation and emission slit widths $=5 \mathrm{~nm}$ ). The fluorescence intensity at $329 \mathrm{~nm}$, the wavelength with the greatest difference in signal intensity across the range of urea concentrations investigated, was plotted versus urea concentration to allow comparison between the wild-type enzyme and site-directed variants (Walters et al. 2009). 
2.4 Results

\subsubsection{Construction and Purification of eCBL Variants}

The K5D, K17D, D37K, D382K, K5/D382 and K17D/D37K charge-reversal variants of eCBL were produced by overlap-extension polymerase chain reaction (OE-PCR). The products of the second OE-PCR product (Figure 2.4) were introduced between the NdeI and BamHI sites of the pTrc3 vector. The site-directed variants were expressed and purified via Ni-NTA affinity chromatography and enzyme fractions assessed as $\geq 95 \%$ purity by SDS-PAGE, were pooled, concentrated and stored at $-80^{\circ} \mathrm{C}$ (Figure 2.5). The $\mathrm{K} 17 \mathrm{D}$ and $\mathrm{D} 382 \mathrm{~K}$ variants could not be purified. The yields of the other six variants ranged between 0.38 and $1.96 \mathrm{mg}$ of protein per litre of culture (Table 2.2). 


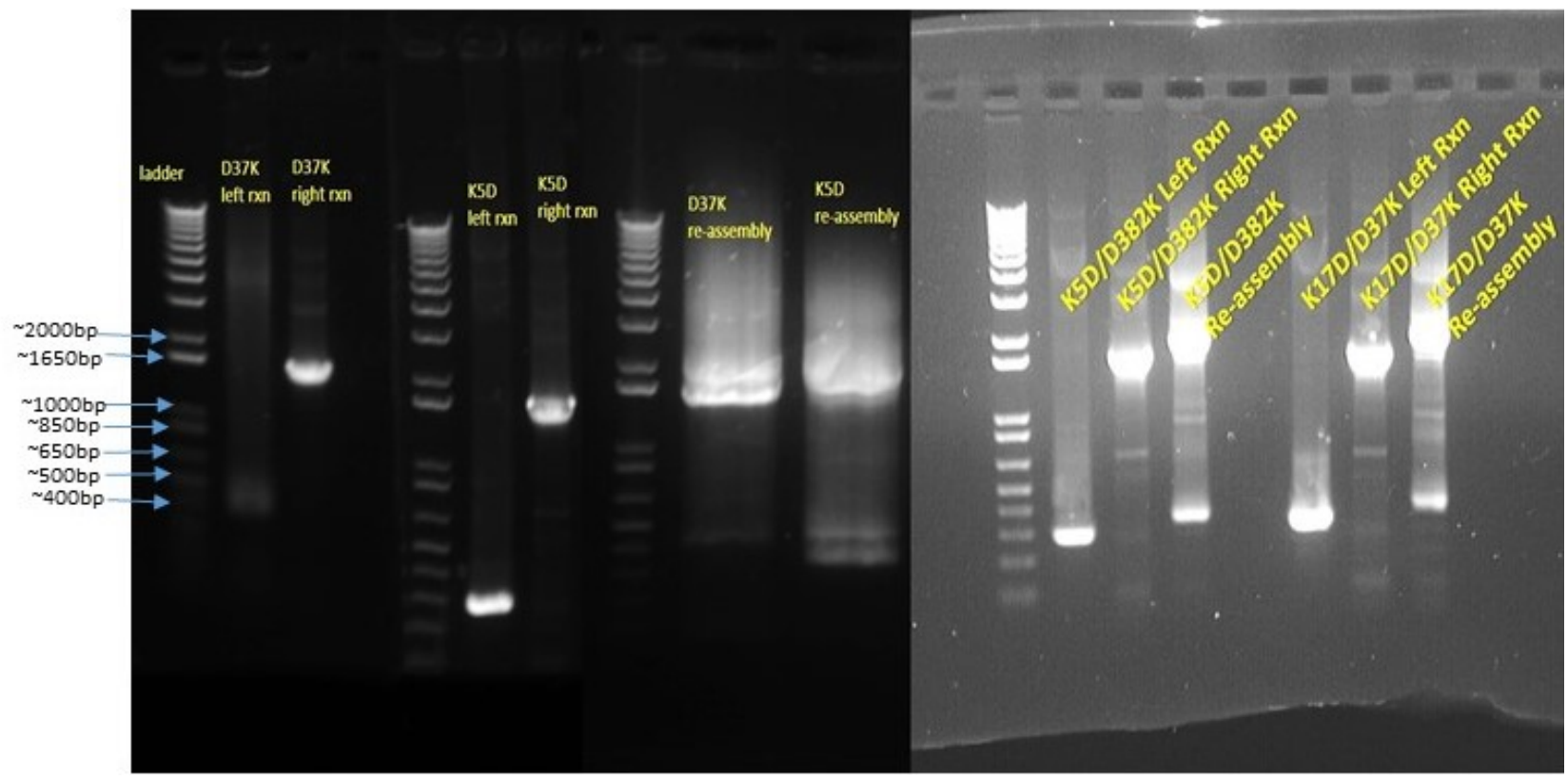

Figure 2.4: Agarose (1\%) gel of the 3' and 5' amplicons, labelled 'left rxn' and 'right rxn', respectively, for each site-directed mutant as well as the re-assembly products of the second PCR step in the construction of the site-directed mutants, via the overlap-extension method. 


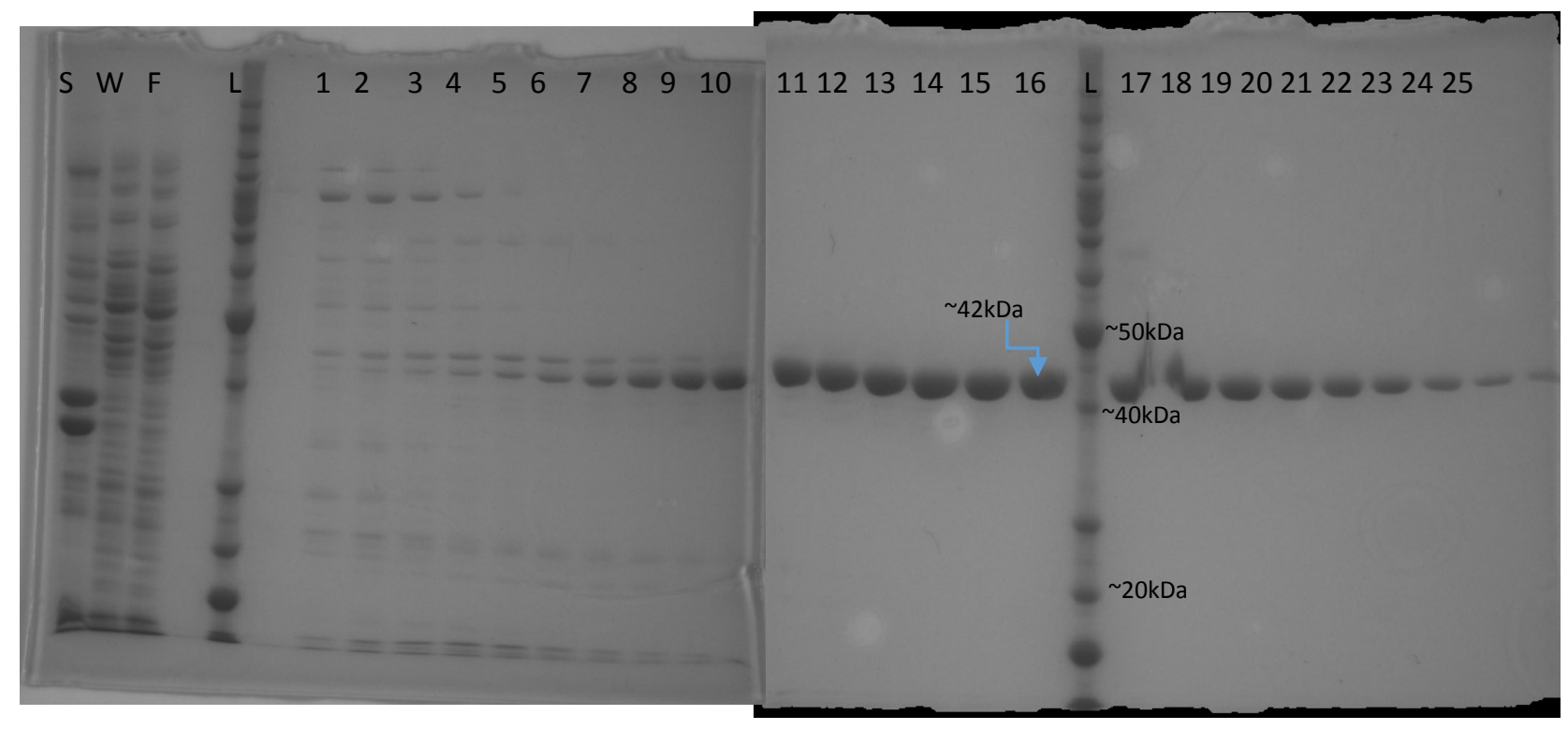

Figure 2.5: The SDS-PAGE gels, stained with Comassie Blue, of the purification of eCBL-K5D. Lanes are designated as $\mathrm{S}=$ crude lysate, $\mathrm{W}=$ wash with buffer $\mathrm{A}, \mathrm{F}=$ flow through and $\mathrm{L}=$ Ladder. Fractions in lanes 11-26 were pooled and concentrated.

Table 2.2: site directed variants and their protein yield

\begin{tabular}{|cc|}
\hline eCBL variants & Yield $(\mathbf{m g} / \mathbf{L})$ \\
& \\
\hline $\mathrm{K} 5 \mathrm{D}$ & 0.38 \\
\hline $\mathrm{D} 37 \mathrm{~K}$ & 1.96 \\
\hline $\mathrm{K} 5 \mathrm{D} / \mathrm{D} 382 \mathrm{~K}$ & 1.28 \\
\hline $\mathrm{K} 17 \mathrm{D} / \mathrm{D} 37 \mathrm{~K}$ & 0.68 \\
\hline
\end{tabular}




\subsubsection{Kinetic Characterization of the Site-Directed Variants}

The kinetic parameters of the eCBL variants K5D, D37K, K17D/D37K and K5D/D382K were determined and compared with those of the wild-type enzyme (Table 2.3). The catalytic efficiency of the wild-type enzyme is within 2-fold of the value reported by Lodha et al. (2010). The activity of the K17D and D382K variants could not be assessed as it was not possible to purify these proteins. The $\mathrm{K} 5 \mathrm{D}$ and $\mathrm{D} 37 \mathrm{~K}$ single-substitution variants both display a 13 -fold reduction in catalytic efficiency that is dominated by 16 and 11 -fold decreases in $\mathrm{k}_{\text {cat }}$, respectively, compared to the wild-type eCBL. In contrast, the $\mathrm{K}_{\mathrm{m}}$ of both variants is unchanged, within experimental error (Table 2.3). Similarly, the catalytic efficiency of the eCBL$\mathrm{K} 17 \mathrm{D} / \mathrm{D} 37 \mathrm{~K}$ and $\mathrm{K} 5 \mathrm{D} / \mathrm{D} 382 \mathrm{~K}$ double-substitution variants, which maintain the charged pair but swap the charges, is reduced by 20 and 11-fold and these changes are dominated by 21 and 12fold decreases in $\mathrm{k}_{\mathrm{cat}}$, respectively.

\subsubsection{Urea Denaturation of wt-eCBL and eCBL Mutants}

The fluorescence-monitored, urea denaturation of the wild-type eCBL enzyme exhibits a multistate unfolding profile with transition midpoints of $\mathrm{C}_{\mathrm{m} 1} \approx 1.5 \mathrm{M}, \mathrm{C}_{\mathrm{m} 2} \approx 3.5 \mathrm{M}, \mathrm{C}_{\mathrm{m} 3} \approx 5 \mathrm{M}$ and $\mathrm{C}_{\mathrm{m} 4}$ $\approx 6 \mathrm{M}$ (Figure 2.6) (Jaworski et al., 2013). The eCBL-K5D variant shows a reduction of $\sim 1 \mathrm{M}$ urea for each of the transition phases in addition to a lower fluorescence intensity of the peak at $2 \mathrm{M}$ urea, compared to the wild-type (Figure 2.7). The denaturation profile of eCBL-D37K is similarly affected although the transitions are reduced by $\sim 0.5 \mathrm{M}$ urea (Figure 2.8 ). In contrast, the K5D/D382K and K17D/D37K double-substitution variants exhibit similar profiles as wildtype eCBL, with transitions occurring at only 0.1-0.2 M lower urea concentration (Figures 2.9 and 2.10). 
Table 2.3: Steady state kinetic parameters of wild-type eCBL and theK5D, K17D, D37K and D382K, K5D/D382K and K17D/D37K variants. ${ }^{a}$

\begin{tabular}{|l|c|c|c|}
\hline Enzyme & $\mathbf{K}_{\mathbf{c a t R}}\left(\mathbf{s}^{\mathbf{- 1}}\right)$ & $\mathbf{K}_{\mathbf{m R}}{ }^{\mathbf{L}-\mathbf{C t h}}(\mathbf{m M})$ & $\mathbf{K}_{\mathbf{c a t}} / \mathbf{K}_{\mathbf{m R}}{ }^{\mathbf{L}-\mathbf{C t h}}\left(\mathbf{M}^{\mathbf{- 1}} \mathbf{s}^{\mathbf{- 1}}\right)$ \\
\hline Wt eCBL & $44 \pm 1$ & $0.14 \pm 0.02$ & $(3.2 \pm 0.3) \times 10^{5}$ \\
\hline Wt eCBL $^{b}$ & $34.1 \pm 0.6$ & $0.18 \pm 0.01$ & $(1.9 \pm 0.1) \times 10^{5}$ \\
\hline eCBL-K5D & $2.8 \pm 0.1$ & $0.12 \pm 0.02$ & $(2.3 \pm 0.3) \times 10^{4}$ \\
\hline eCBL-D37K & $4 \pm 1$ & $0.18 \pm 0.03$ & $(2.4 \pm 0.3) \times 10^{4}$ \\
\hline eCBL-K17D/D37K & $2.06 \pm 0.06$ & $0.13 \pm 0.02$ & $(1.6 \pm 0.2) \times 10^{4}$ \\
\hline eCBL-K5D/D382K & $3.8 \pm 0.1$ & $0.13 \pm 0.02$ & $(2.9 \pm 0.3) \times 10^{4}$ \\
\hline
\end{tabular}

${ }^{a}$ Kinetic parameters for hydrolysis of L-Cth were determined at $25^{\circ} \mathrm{C}$ in $50 \mathrm{mM}$ Tris buffer $(\mathrm{pH}$ 8.5) containing 20 $\mu \mathrm{M}$ PLP, $2 \mathrm{mM}$ DTNB, 0.004- 6.2 $\mathrm{mM}$ L-Cth and 0.675-2.17 $\mu \mathrm{M}$ eCBL. Data were fit to equation 2.1 and 2.2.

${ }^{b}$ Values of kinetic parameters are from Lodha et al., 2010 


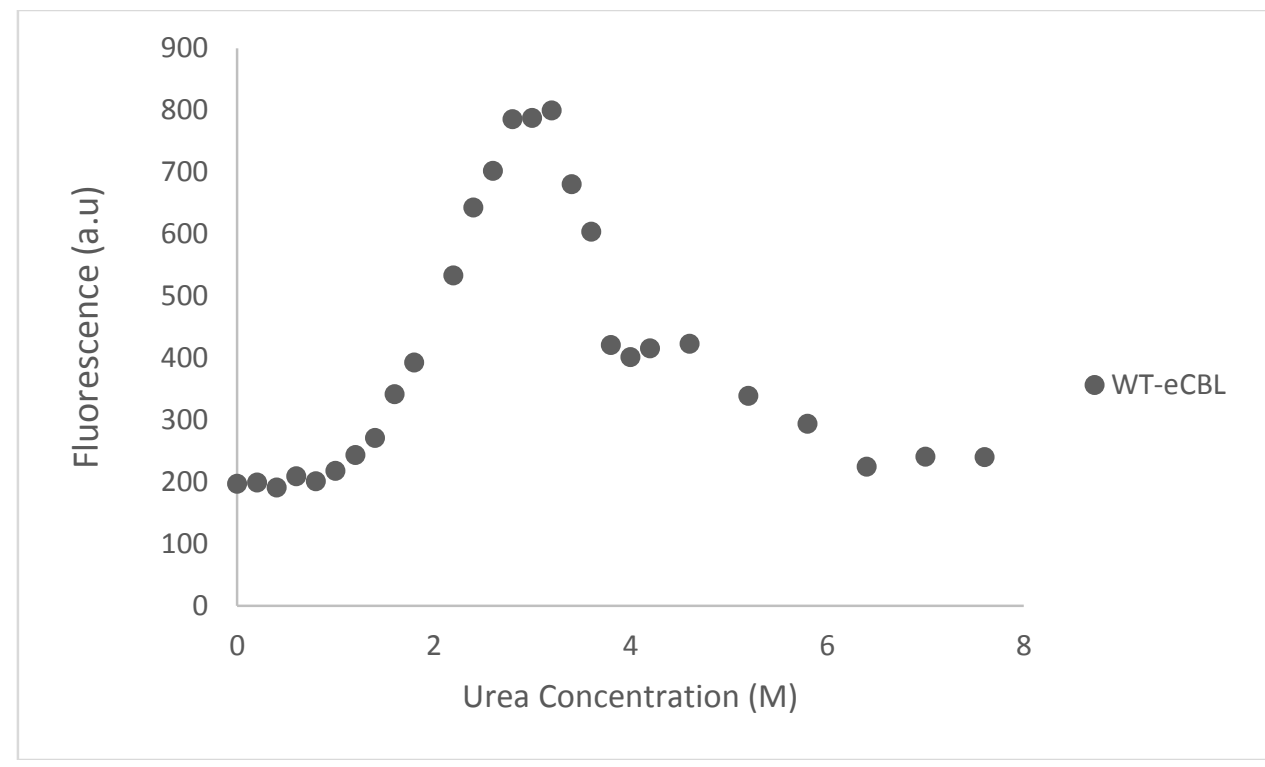

Figure 2.6: The dependence of the tryptophan emission spectrum of $0.5 \mu \mathrm{M}$ wt-eCBL on urea concentration. Spectra were recorded between 300 and $380 \mathrm{~nm}\left(\lambda_{\mathrm{ex}}=295 \mathrm{~nm}\right.$, excitation and emission slit $=5 \mathrm{~nm})$.

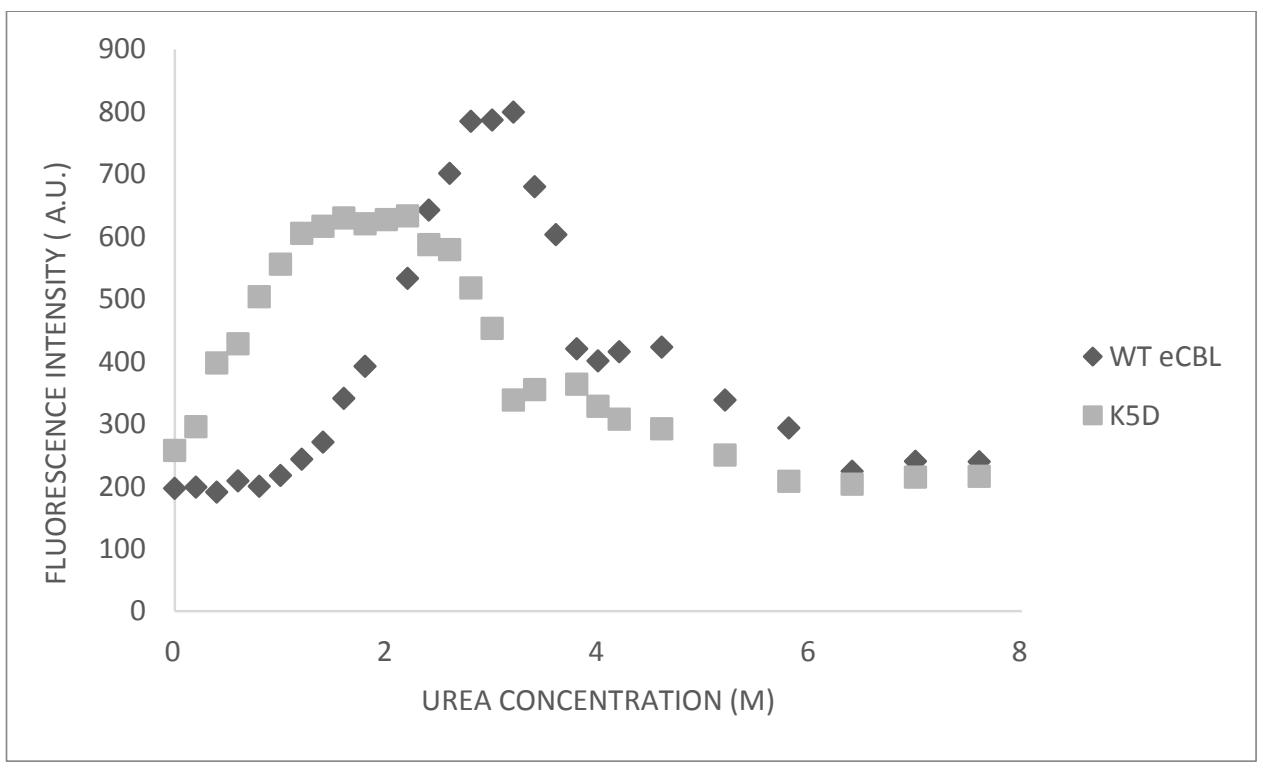

Figure 2.7: Comparison of the dependence of the tryptophan emission spectrum of $0.5 \mu \mathrm{M}$ wildtype and K5D eCBL on urea concentration. Spectra were recorded between 300 and $380 \mathrm{~nm}\left(\lambda_{\mathrm{ex}}=\right.$ $295 \mathrm{~nm}$, excitation and emission slit $=5 \mathrm{~nm})$. 


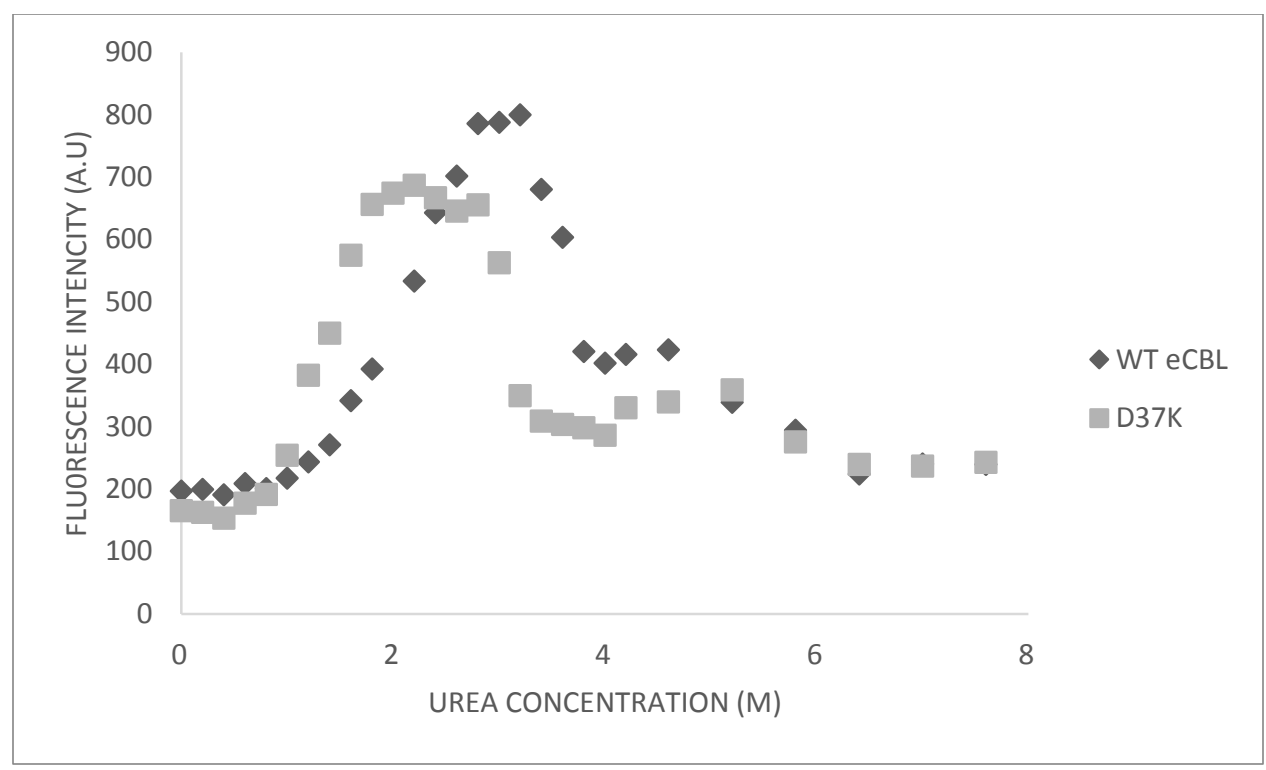

Figure 2.8: Comparison of the dependence of the tryptophan emission spectrum of $0.5 \mu \mathrm{M}$ wildtype and D37K eCBL on urea concentration. Spectra were recorded between 300 and 380nm $\left(\lambda_{\mathrm{ex}}=295 \mathrm{~nm}\right.$, excitation and emission slit $\left.=5 \mathrm{~nm}\right)$.

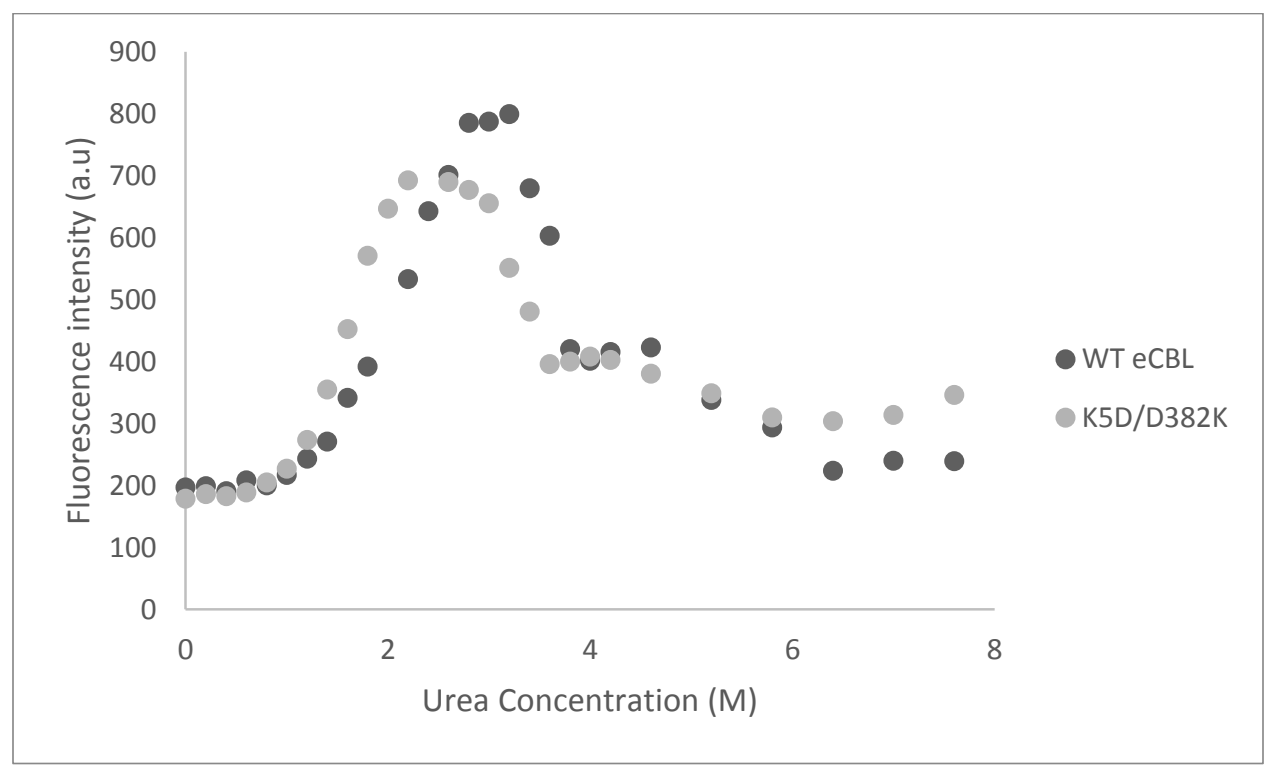

Figure 2.9: Comparison of the dependence of the tryptophan emission spectrum of $0.5 \mu \mathrm{M}$ wildtype and K5D/D382K eCBL on urea concentration. Spectra were recorded between 300 and $380 \mathrm{~nm}\left(\lambda_{\mathrm{ex}}=295 \mathrm{~nm}\right.$, excitation and emission slit $\left.=5 \mathrm{~nm}\right)$. 


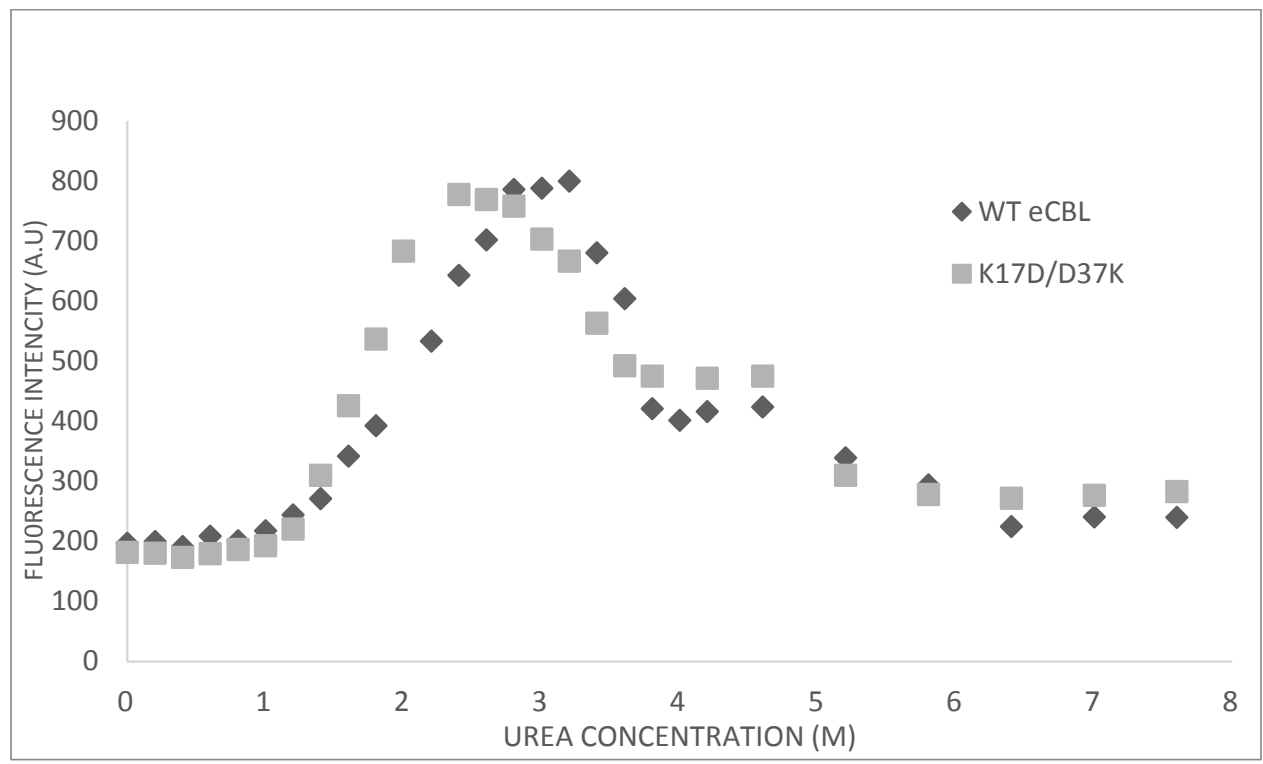

Figure 2.10: Comparison of the dependence of the tryptophan emission spectrum of $0.5 \mu \mathrm{M}$ wild-type and K17D/D37K eCBL on urea concentration. Spectra were recorded between 300 and $380 \mathrm{~nm}\left(\lambda_{\mathrm{ex}}=295 \mathrm{~nm}\right.$, excitation and emission slit $\left.=5 \mathrm{~nm}\right)$. 


\subsection{Discussion}

The $\beta$-elimination of L-cystathionine catalyzed by CBL hydrolyzes the cystathionine produced by CGS, in the first step of the transsulfuration pathway of bacteria and plants. The homotetrameric eCBL associates as a dimer of catalytic dimers, in which each of the two monomers contributes residues to both active sites of the dimer, such that the enzyme possesses four active sites. While studies from our lab have demonstrated the role of monomer-monomer interactions within each dimer pair in forming a catalytically competent active site, the role of interactions with the opposing dimer have not been reported (Lodha et al., 2010; Lodha and Aitken, 2011; Manders et al., 2013). The dimer interface of eCBL is characterized by hydrogenbonding and salt-bridge interactions and much of the interface is comprised of residues of helices 1 and 2 of the N-terminal domain and helices 12 and 16 of the C-terminal domain (Figure 2.2) (Clausen et al., 1996). This study investigated the effect of charge reversal of individual residues (K5D, K17D, D37K and D382K) as well as interacting pairs (K5D/D382K and K17D/D37K) and focused on two salt-bridges situated at the periphery of the dimer interface. The results contribute to our understanding of the factors that underlie active-site architecture and dynamics by establishing the influence of interactions at the dimer interface.

Two of the residues targeted in this study (K5 and D382) are within helices 1 (residues 5-12) and 16 (residues 380-395) that stabilize tetramer formation. These residues span the dimer interface via four hydrogen bonds, including water-mediated interactions. Another interface-bridging interaction occurs between the segment including the short helix 2 (residues 17-20), containing K17, and D37 of the extended loop, immediate C-terminal of this helix of the associated symmetry mate in the monomer of the opposing dimer pair (i.e. K17 of one monomer interacts with D37 of a monomer in the other catalytic dimer) (Figure 2.2). Together the K5-D382 and 
K17-D37 residues pairs form the two outermost salt bridges of the eCBL dimer interface (Clausen et al., 1996). Residue D37 is also located in one of the two segments that adopt distinct conformations, between eCBL and $\mathrm{yCGL}$, at the mouth of the active site and, as such, may influence reaction specificity (Clausen et al., 1998; Messerschmidt et al., 2003; Manders et al., 2013).

Individual substitution variants were constructed to probe the effect of disrupting each salt bridge by inverting the charges of one of the partner residues to produce either two negative or two positive proximal charges. In contrast, the double substitutions maintain the overall charge neutrality of the residue pair as the side chains of aspartate and lysine are negatively and positively charged, respectively, at physiological $\mathrm{pH}$. Destabilizing effects of the doublesubstitution variants is expected to result from interactions between each residue and others in their immediate environment and, as such, the effect of these substitutions on enzyme stability was expected to be less than that of the single substitutions. This expectation is fulfilled by the observation that while the $\mathrm{K} 5 \mathrm{D} / \mathrm{D} 382 \mathrm{~K}$ and $\mathrm{K} 17 \mathrm{D} / \mathrm{D} 37 \mathrm{~K}$ variants could be purified, only two of the four single substitution variants (K5D and D37K) are stable enough to be soluble. This demonstrates that the effect of co-locating a pair of positive (D382K) or negative charges (K17D) was sufficiently destabilizing as to preclude formation of a stable tetramer and suggests that, despite the polar nature of the dimer interface, individual dimers are not stable in solution. The yield obtained for the site- directed variants $(0.38-1.96 \mathrm{mg}$ of protein/ litre of culture) was 11-57-fold lower than the yield of $22 \mathrm{mg}$ of protein per litre of media reported by Farsi and colleagues, suggesting that the charge-reversal substitutions reduce enzyme stability (Farsi et al., 2009) 
Tryptophan residues are sensitive probes of protein conformation as the spectrum of a solvent exposed tryptophan is red-shifted approximately $20 \mathrm{~nm}$ to $\sim 350 \mathrm{~nm}$, compared to the same residue when it is shielded from solvent $(\sim 330 \mathrm{~nm})$ within a folded protein. The fluorescencemonitored, urea denaturation of wild-type eCBL has been thoroughly characterized (Jaworski et al., 2013). Therefore, this method was employed to assess the effect of the charge-reversal substitutions on the denaturation profile of eCBL. As anticipated, the K5D and D37K variants decrease the midpoint in all transitions, and particularly the primary $\mathrm{C}_{\mathrm{m} 1}$ and $\mathrm{C}_{\mathrm{m} 2}$, by $0.5-1 \mathrm{M}$ urea (Figures 2.7 and 2.8) while the destabilizing effect of both double-substitution variants is only 0.1-0.2M urea (Figures 2.9 and 2.10).

Each eCBL monomer contains six tryptophans (W131, W188, W230, W276, W300 and W340). Residues W188 and W340 are situated in the active site cleft, at the interface between the PLPbinding and C-terminal domains, and W188 provides a particularly useful probe of active site conformation as, upon release from quenching, it exhibits hyper-fluorescence and dominates the fluorescence spectrum of eCBL (Figure 2.6) (Jaworski and Aitken, 2013). Jaworski and Aitken proposed that the observed hyper-fluorescence of W188, upon release from quenching by $2 \mathrm{M}$ urea, is the result of reorientation of W188 and indicates increased active site flexibility as the enzyme approaches a molten globule state (Jaworski and Aitken, 2013). Therefore, we anticipated that shifts in the $0-2 \mathrm{M}$ transition zone of the charge-reversal substitutions would parallel the magnitude of their impact on the kinetic parameters. However, the 13-fold reduction in $\mathrm{k}_{\text {cat }} / \mathrm{K}_{\mathrm{m}}$ for both $\mathrm{K} 5 \mathrm{D}$ and $\mathrm{K} 37 \mathrm{~K}$ is of similar magnitude as the 21 and 11 -fold decreases observed for the K17D/D37K and K5D/D382K double-substitution variants. In all cases, the 1121 -fold decrease in catalytic efficiency is dominated by a similar reduction in turnover $\left(\mathrm{k}_{\mathrm{cat}}\right)$, while the effect on $\mathrm{K}_{\mathrm{m}}$ is negligible (Table 2.3). The similarity in the effect of the single and 
double substitution variants on the kinetic parameters, despite the greater impact of the former on the denaturation profile and stability of eCBL, demonstrates the exquisite balance of the structure-function relationships that maintain the active site in a catalytically competent conformation.

The K5/D382 interaction positions the C-terminal $\alpha$-helix (residues 380-395) with respect to the opposing catalytic dimer, tethering the N-terminus of this helix, which packs between a pair of $\alpha$ helices that stabilize the four-stranded $\beta$-sheet that forms the structural core of the $\mathrm{C}$-terminal domain. Helix 16 interacts directly, along its length, with the helix formed by residues 320-330, from which extends the loop containing key active-site residues S339 and W340. Residue S339 plays a primary role in tethering and positioning the catalytic base, while W340 interacts with both the O3' moiety of the PLP cofactor and the $\alpha$-carboxylate group of the substrate, thereby regulating their relative positions. The $\mathrm{C}$-terminal helix also interacts directly, along its length, with the central $\beta$-strand (residues 370-374) of the antiparallel $\beta$-sheet. Residue R372, which plays an essential role in coordinating the $\alpha$-carboxylate moiety of the substrate, is centrally situated within this $\beta$-strand (Clausen et al., 1996). Previous studies from our lab have shown that substitution of S339 or R372 to eliminate the hydrogen-bonding capacity of the side chain and preclude its role in the active site reduces catalytic efficiency by at least three orders of magnitude (Lodha et al., 2010; Lodha and Aitken, 2011). Therefore, even the subtle structural change resulting from charge reversal of the K5D/D382K and K17D/D37K double-substitution variants may be translated to the active site via these interactions.

The results of this study confirm the proposed role of the K5/D382 and K17/D37 charged interactions in maintaining and stabilizing the quaternary structure of eCBL. We have also demonstrated that the conformational changes resulting from charge-reversal substitutions of 
these residues are translated to the active site. The observed reductions in catalytic efficiency and in the transition, of the fluorescence-monitored urea denaturation profile of eCBL, corresponding to release from quenching of W188, suggest that modification of these dimer-interface salt bridges alters the architecture and/or dynamics of the active site. Interestingly, the K5-D382 salt bridge is conserved in yCGL, while the K17-D37 interaction is replaced by a pair of hydrophobic residues (Messerschmidt et al., 2003). Future studies following up this work could explore the role of the corresponding residues in yCGL and investigate the effect of the interactions that stabilize the dimer interface on the reaction specificity of these enzymes, which catalyze distinct hydrolysis reactions of the same pseudosymmetric substrate. 
Chapter 3: Interconversion of a pair of residues in the active sites of cystathionine $\beta$-lyase and cystathionine $\gamma$-lyase 


\subsection{Abstract}

Cystathionine $\beta$-lyase (CBL) catalyzes the PLP-dependent $\beta$-elimination of L-cystathionine, yielding L-homocysteine, pyruvate and ammonia, while the $\gamma$-elimination of this pseudosymmetric substrate by cystathionine $\gamma$-lyase (CGL) produces L-cysteine, $\alpha$-ketobutyrate and ammonia. This distinct reaction specificity requires that the L-cystathionine substrate be bound in opposite orientations by the two enzymes and that the lysine residue that acts as the catalytic base, upon release from Schiff base linkage with the PLP cofactor, be differentially tethered by interaction with nearby residues. Comparison of the structures of Escherichia coli CBL (eCBL) and Saccharomyces cerevisiae CGL, and of eCBL in the resting state and in complex with the inhibitor aminoethoxyvinylglycine suggests that conserved active site residues S50 and S200 of yCGL may participate in guiding the $\varepsilon$-amino group of the catalytic base (yCGL-K203) to ensure the appropriate proton transfers are accomplished. These residues, and the corresponding G57 and A207 of Escherichia coli CBL (eCBL), were targeted for interconversion to probe the role(s) of the hydroxyl moieties of the conserved yCGL residues. The catalytic efficiency $\left(\mathrm{k}_{\mathrm{cat}} / \mathrm{K}_{\mathrm{m}}\right)$ of the eCBL G57S and A207S variants and the yCGL S50A, S50G and S200A variants is reduced by only 5-11-fold, compared to the respective wild-type enzymes. These results suggest that the residues investigated are not primary determinants in regulating the mobility of the catalytic base or in maintaining a catalytically competent active site architecture and likely do not regulate reaction specificity. 


\subsection{Introduction}

The possibility of modifying the substrate and/or reaction specificity or the temperature, $\mathrm{pH}$ or solvent stability of enzymes presents opportunities to increase yield and energy efficiency. This could be effective in a range of industrial processes by introducing these powerful biocatalysts. The PLP-dependent enzymes of the transsulfuration pathways participate in the interconversion of sulfur-containing amino acids of major biological and industrial importance (Farsi et al., 2009). The ability of these closely related enzymes to catalyze distinct transformations of similar or identical substrates provides an ideal model system for studies exploring the molecular mechanisms that underlie the specificity of enzymes reliant on this catalytically versatile cofactor, with the goal of engineering PLP-dependent enzymes for biotechnological applications (Hopwood et al., 2014).

In the bacterial transsulfuration pathway, cystathionine $\gamma$-synthase (CGS) replaces the succinyl moiety of $O$-succinylhomocysteine with L-cysteine, forming L-cystathionine, which is subsequently cleaved by cystathionine $\beta$-lyase (CBL) to yield L-homocysteine. The reverse transsulfuration pathway of yeast and mammals comprises the condensation of L-serine and Lhomocysteine by cystathionine $\beta$-synthase (CBS), forming the common L-cystathionine central metabolite, which is hydrolyzed by cystathionine $\gamma$-lyase (CGL) to produce L-cysteine (Aitken and Kirsch, 2005). The cystathionine-cleaving enzymes CBL and CGL are both members of the $\gamma$-subfamily of fold-type I of PLP-dependent enzyme and along with other enzymes of this family, share approximately $20-40 \%$ amino acid identity. These enzymes are very similar in structure, as illustrated by the rmsd of $\sim 1.5 \AA$ for the superposition of approximately $350 \mathrm{C} \alpha$ atoms of the protein backbone (Steegborn et al., 1999; Aitken and Kirsh, 2005; Messerchmidt et 
al., 2003). The cystathionine intermediate, common to both pathways, is cleaved via distinct $\beta$ and $\gamma$-elimination reactions by CBL and CGL, respectively. (Farsi et al., 2009). This requires that the pseudosymmetric cystathionine substrate bind to the active sites of these two enzymes in distinct orientations (Messerchmidt et al., 2003).

The active sites of fold-type I enzymes, including those of the transsulfuration pathways (CBL, CGL and CGS), are comprised of residues from each of monomers from the catalytic dimer (Clausen et al., 1996; Messerschmidt et al., 2003). Many of the residues lining the active site, and playing central roles in substrate binding and catalysis, are conserved between CBL, CGL and CGS. Previous studies from our lab have led to the theory that substrate and reaction specificity are regulated by subtle differences in active site architecture that regulate both the orientation of substrate binding, with respect to the cofactor and catalytic residue(s), and the mobility of the latter (Hopwood et al., 2014; Manders et al., 2013; Jaworski et al., 2012; Lodha and Aitken 2011; Aitken et al., 2011; Lodha et al., 2010). Identification of the key structurefunction relationships underlying these differences will unlock the potential for modifying the specificity of these enzymes. The ability of the structurally conserved CBL and CGL enzymes to catalyze distinct hydrolysis reactions of the same substrate provides a useful system to explore these factors (Clausen et al., 1996; Messerchmidt et al., 2003; Aitken et al., 2011).

One of the most striking differences between the active sites of the transsulfuration enzymes catalyzing $\beta$ - and $\gamma$-elimination/replacement reactions is the replacement of a pair of conserved aromatic residues (F55 and Y338 of Escherichia coli CBL, eCBL) in the former by those with acidic side chains (E48 and E333 of Saccharomyces cerevisiae CGL, yCGL and D45 and E325 of Escherichia coli CGS, eCGS) in the latter. On the basis of structural comparison, these residues were proposed as primary determinants of specificity (Clausen et al., 1998; 
Messerschmidt et al., 2003). However, Farsi and colleagues demonstrated that while these residues may play a role as determinants of specificity, likely by influencing active site architecture, they are not sufficient as their interconversion reduced the catalytic efficiency but did not modify reaction specificity (Farsi et al., 2009). This suggests that multiple residues likely act in concert to regulate specificity. Both of the residues investigated by Farsi et al. are situated immediately adjacent to residues (Y56 and S339 of eCBL; Y49 and S334 of yCGL) that have been shown to tether the $\varepsilon$-amino group of the catalytic base (eCBL-K210), following the transaldimination reaction in which the substrate replaces this lysine residue in Schiff-base linkage with the PLP cofactor (Farsi et al., 2009; Lodha and Aitken, 2011; Jaworski et al., 2013). The importance of the side-chain hydroxyl of eCBL-S339 is demonstrated by the reduction of four orders of magnitude in catalytic efficiency observed upon replacement of this residue with alanine. Residue eCBL-Y56 forms hydrogen bonds to both the phosphate moiety of the cofactor and the $\varepsilon$-amino group of the catalytic base and substitution with phenylalanine, which removes these interactions demonstrated that while this tyrosine residue is involved in positioning the catalytic base, its role is secondary to S339, in the context of eCBL (Lodha and Aitken, 2011). Manders and colleagues subsequently investigated residues S32 and S33, which interact with S339 in eCBL, and observed that their replacement with alanine had negligible effect on eCBL activity (Manders et al., 2013). Seeking alternate residues that might differentially influence the positioning of the $\varepsilon$-amino group of the active site lysine, and thereby influence $\beta$ versus $\gamma$ elimination specificity, we compared the structures of eCBL and yCGL as well as of eCBL in the presence and absence of the inhibitor aminoethoxyvinylglycine (AVG), which forms an external aldimine with the cofactor, thereby releasing the catalytic lysine. Similar inhibitor bound structures of yCGL are not available and the resting state structure of this enzyme is of lower 


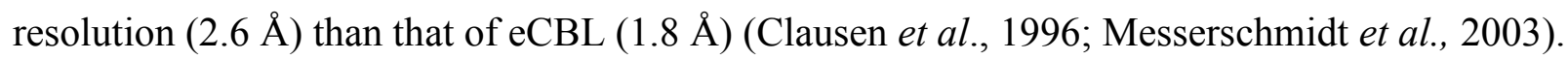
Residues G57 and A207 of eCBL, conserved as serine residues (S50 and S200 of yCGL) in CGL, were selected for exploration in this study in order to assess and compare their role in the $\beta$-versus $\gamma$-eliminations of cystathionine catalyzed by eCBL and yCGL, respectively. A direct role for yCGL-S200 and an indirect role for S50, mediated by the adjacent Y49, in influencing the positioning of the $\varepsilon$-amino group of the active site lysine were postulated. In order to test this hypothesis, a series of substitutions that probe the role of the side-chain hydroxyl moiety of the serine residues of yCGL, in the context of both enzymes, was constructed. It was anticipated that substrate binding would not be affected, unless via an unanticipated effect of the substitutions on active site architecture, while turnover $\left(\mathrm{k}_{\mathrm{cat}}\right)$ would be substantially altered, likely to a greater effect in yCGL than eCBL, by the mispositioning of the catalytic base. 


\subsection{Materials and Methods}

\subsubsection{Reagents}

Ampicillin, 5, 5'-dithio-bis-(2-nitrobenzoic acid) (DTNB) and imidazole were purchased from Fisher Scientific while, L-cystathionine was from Sigma.. Restriction endonucleases, Antarctic phosphatase and T4 DNA ligase were obtained from New England Biolabs. Nickelnitrilotriacetic acid (Ni-NTA) resin was acquired from Qiagen. Oligonucleotide primers were synthesized by Integrated DNA Technologies and site-directed mutants were sequenced by BioBasic.

\subsubsection{Construction of Site-Directed Mutants}

Overlap-extension polymerase chain reaction (PCR) with the pSECseq0 (GGC GTC AGG CAG CCA TCG GAA GCT G) and pSECseq7r (GCC CGC CAC CCT CCG GGC CGT TGC TTC GC) primers (which flank the multiple cloning site of the vector) and the respective mutagenic primers was employed to construct the S200A, S50A and S50G variants of yCGL, and G57S and A207S variants of eCBL. The resulting amplicons were gel purified prior to insertion between the NdeI and BamHI sites or the NdeI and Xbal sites of the pTrc3 plasmid, for eCBL and yCGL, respectively. The pTrc3 plasmid encodes an N-terminal, 6-His affinity tag, which enables purification of the recombinant protein using immobilized metal ion affinity chromatography. The restriction-digested $\mathrm{p} \operatorname{Trc} 3$ vector was treated with Antarctic phosphatase prior to ligation, to reduce background due to reannealing of the digested vector. The pTrc3-eCBL and pTrc3-yCGL constructs were transformed into $E$. coli ER1821 metC::cat strain in which the coding sequence 
of eCBL is replaced by that of chloramphenicol acetyltransferase; thereby, precluding contamination with the wild-type E. coli enzyme (Farsi et al., 2009). The full coding regions and plasmid junctions of each site-directed mutant were sequenced by BioBasic prior to expression and purification to ensure only the desired mutation(s) were present. 
Table 3.1: site-directed mutants and their respective mutagenic primers ${ }^{a}$

\begin{tabular}{|l|l|}
\hline $\begin{array}{l}\text { eCBL } \\
\text { variants }\end{array}$ & Primer Sequence \\
\hline G57S* & 5'-gagttgttctatagccggcgcggaacg \\
\hline A207S* & 5'-gtttctattcaaagcgccaccaaatatc \\
\hline $\begin{array}{l}\text { yCGL } \\
\text { variants }\end{array}$ & Primer sequence \\
\hline S50A* & 5'-cttacgaatacgcgagatctcaaaatc \\
\hline S50G* & 5'-cttacgaatacggcagatctcaaaatc \\
\hline S200A* & 5'-gacatcgttgtccacgcggctacaaagtac \\
\hline
\end{tabular}

${ }^{a}$ The second mutagenic primer is the reverse compliment of the primer listed in the table 


\subsubsection{Expression and Purification of the Site-Directed Variants}

Overnight cultures of $100 \mathrm{ml}$ of wild-type or site-directed mutants of yCGL or eCBL were grown in rich growth media $(50 \mathrm{mM}$ potassium phosphate and Vogel Bonner salts Vogel and Bonner, 1956 and containing $7.2 \mathrm{~g}$ tryptone and $14.4 \mathrm{~g}$ yeast extract per liter) containing 50 $\mu \mathrm{g} / \mathrm{mL}$ each of ampicillin and chloramphenicol (Vogel and Bonner, 1956). Three Fernbach flasks, each containing $1 \mathrm{~L}$ of rich media (3x 1L), were inoculated with $20 \mathrm{ml}$ of overnight culture and incubated at $37^{\circ} \mathrm{C}$ and $200 \mathrm{rpm}$ until the $\mathrm{OD}_{600}$ reached 2.5. Expression was induced with $0.15 \mathrm{mM}$ IPTG, followed by incubation for a further 18 hours. The cells were harvested by centrifugation at $5000 \mathrm{rpm}$ for $10 \mathrm{~min}$ and the pellets were washed in $0.85 \% \mathrm{NaCl}$, centrifuged at $5000 \mathrm{rpm}$ for $15 \mathrm{~min}$ and stored at $-80^{\circ} \mathrm{C}$.

The cell pellets were re-suspended in buffer A (50mM potassium phosphate, $\mathrm{pH} 7.8,20 \mu \mathrm{M}$ PLP and $10 \mathrm{mM}$ imidazole), containing $1 \mathrm{mg} / \mathrm{mL}$ of lysozyme, and incubated on ice for $20 \mathrm{mins}$. The cell lysate was sonicated ( 8 cycles of 30 seconds at $50 \%$ duty cycle) and centrifuged at 15000 rpm at $4{ }^{\circ} \mathrm{C}$ for 60 minutes. The resulting supernatant was loaded onto a $1.0 \times 10 \mathrm{~cm}$ column of nickel- nitrilotriacetic acid (Ni-NTA) resin, equilibrated with 20 column volumes of buffer A and the affinity-tagged protein was eluted with a $200-\mathrm{mL}$ linear gradient of $10-200 \mathrm{mM}$ imidazole in buffer A. The fractions collected were assessed for enzyme purity via SDS-PAGE and those containing no visible contaminants were pooled, concentrated and dialyzed against storage buffer (50mM potassium phosphate, $\mathrm{pH} 7.8$, containing $1 \mathrm{mM}$ EDTA, $1 \mathrm{mM}$ dithiotrietol and $20 \mu \mathrm{M}$ PLP). Glycerol was added to $20 \% v / v$ before storage at $-80^{\circ} \mathrm{C}$. The protein concentration of each eCBL and yCGL construct was determined spectroscopically $\left(\varepsilon_{280}=51,105 \mathrm{M}^{-1} \mathrm{~cm}^{-1}\right.$ and 28,880 $\mathrm{M}^{-1} \mathrm{~cm}^{-1}$ for eCBL and yCGL respectively) (Clausen et al. 1997). 


\subsubsection{Determination of Steady State Kinetic Parameters of the Hydrolysis of L-cystathionine}

Kinetic parameters of the site-directed variants were assessed in the context of their ability to complement E.coli strains deficient in eCBL or yCGL and to alter the reaction specificity of the enzyme in comparison to their respective wild-types (eCBL and yCGL). Enzyme activity was measured in a total reaction volume of $100 \mu \mathrm{L}$ at $25^{\circ} \mathrm{C}$ with a Spectramax 390 spectrophometer (Molecular Devices). The assay buffer consisted of 50mM Tris buffer, $\mathrm{pH} 8.5$, or $50 \mathrm{mM}$ potassium phosphate buffer, $\mathrm{pH}$ 7.2, for eCBL and yCGL, respectively. Pyridoxal 5'-phosphate $(2 \mu \mathrm{M})$ was included in the buffer to ensure cofactor saturation of the enzymes assayed. The hydrolysis of L-cystathionine was detected $\left(\varepsilon_{412}=13,600 \mathrm{M}^{-1} \mathrm{~s}^{-1}\right)$ via the reaction of the free thiol of the L-homocysteine (eCBL) or L-cysteine (yCGL) product with 5, 5' - dithiobis- (2nitrobenzoic acid) (DTNB) (Figure 3.1) (Farsi et al., 2009; Ellman, 1959). The assay buffer contained $2 \mathrm{mM}$ DTNB and 0.004-6.2 mM L-cystathionine. A background reading was recorded before initiation of the reaction by the addition of eCBL or yCGL $(0.675-2.17 \mu \mathrm{M}$, depending on the activity of the particular site-directed variant) for all assays. The resulting data was fit to the Michaelis-Menten equation (equation 3.1) to determine $\mathrm{k}_{\mathrm{cat}}$ and $\mathrm{K}_{\mathrm{m}}$ and the catalytic efficiency $\left(\mathrm{k}_{\mathrm{cat}} / \mathrm{K}_{\mathrm{m}}{ }^{\mathrm{L}-\mathrm{Cth}}\right)$ was obtained independently from equation 3.2. Data were fit by nonlinear regression with the SAS software package (SAS Institute, Cary, NC).

$$
\frac{v}{[E]}=\frac{k_{c a t} \times[S]}{K_{m}+[S]}
$$

$$
\frac{v}{[E]}=\frac{k_{c a t} / K_{m} \times[S]}{1+[S] / K_{m}}
$$




\subsection{Results}

\subsubsection{Construction and Purification of eCBL and $y C G L$ Variants}

The eCBL G57S, A207S and yCGL S50A, S50G, S200A variants were produced by overlapextension PCR (Figure 3.1 and 3.2). The reassembled, full-length amplicons of the over-lap extension PCR were introduced into the pTrc3 vector at the $N d e \mathrm{I} / X b a$ l site for the $\mathrm{yCGL}$ constructs and $\mathrm{Ndel} /$ Bam $\mathrm{HI}$ for eCBL constructs. The variants were expressed and purified via Ni-NTA affinity chromatography and enzyme fractions assessed as $\geq 95 \%$ purity by SDS-PAGE, were pooled, concentrated and stored at $-80{ }^{\circ} \mathrm{C}$ (Figure 3.3). The yields of the variants ranged between 2.96-5.21 mg of protein per litre of culture (Table 3.2). 


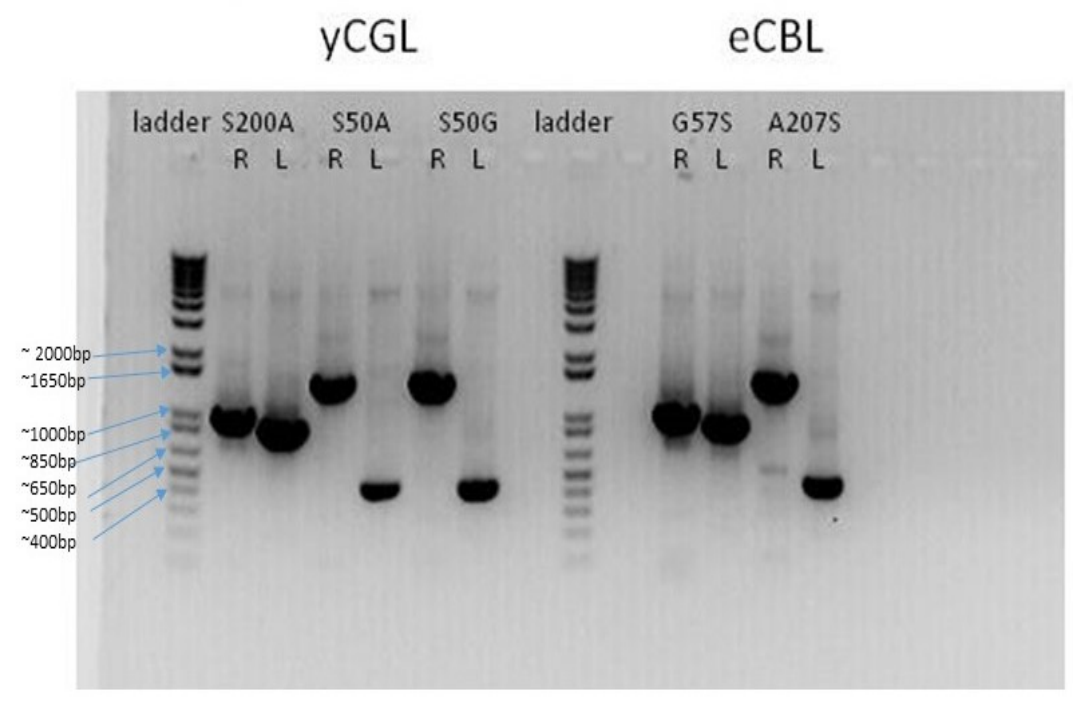

Figure 3.1: Agarose (1\%) gel of the 5' and 3' amplicons, labelled R and L, respectively, for each site-directed mutant.

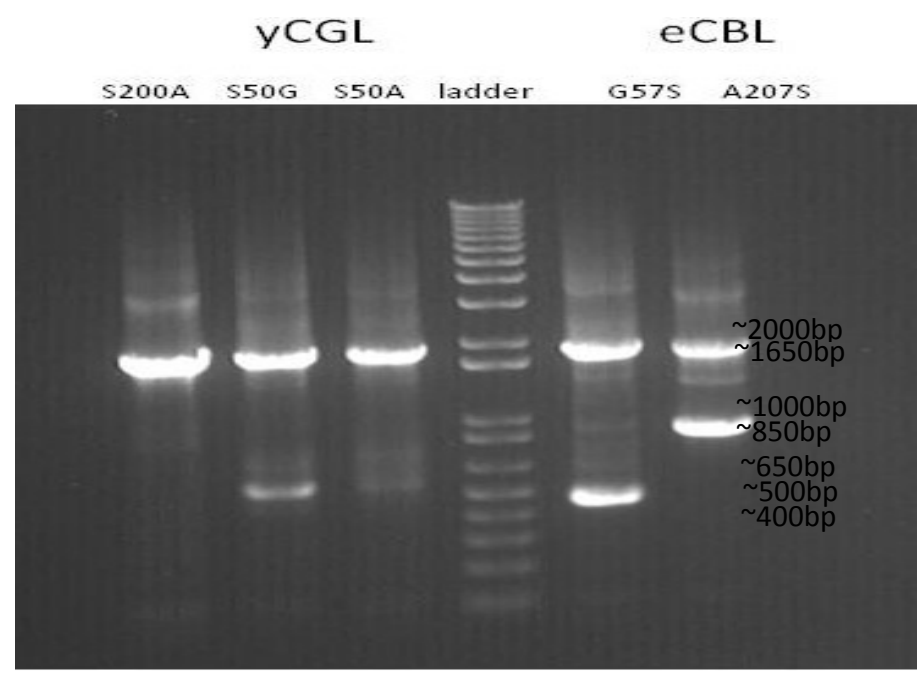

Figure 3.2. Agarose (1\%) gel of the amplification products of the reassembly PCR step in the construction of the site-directed mutants via the overlap-extension method. 


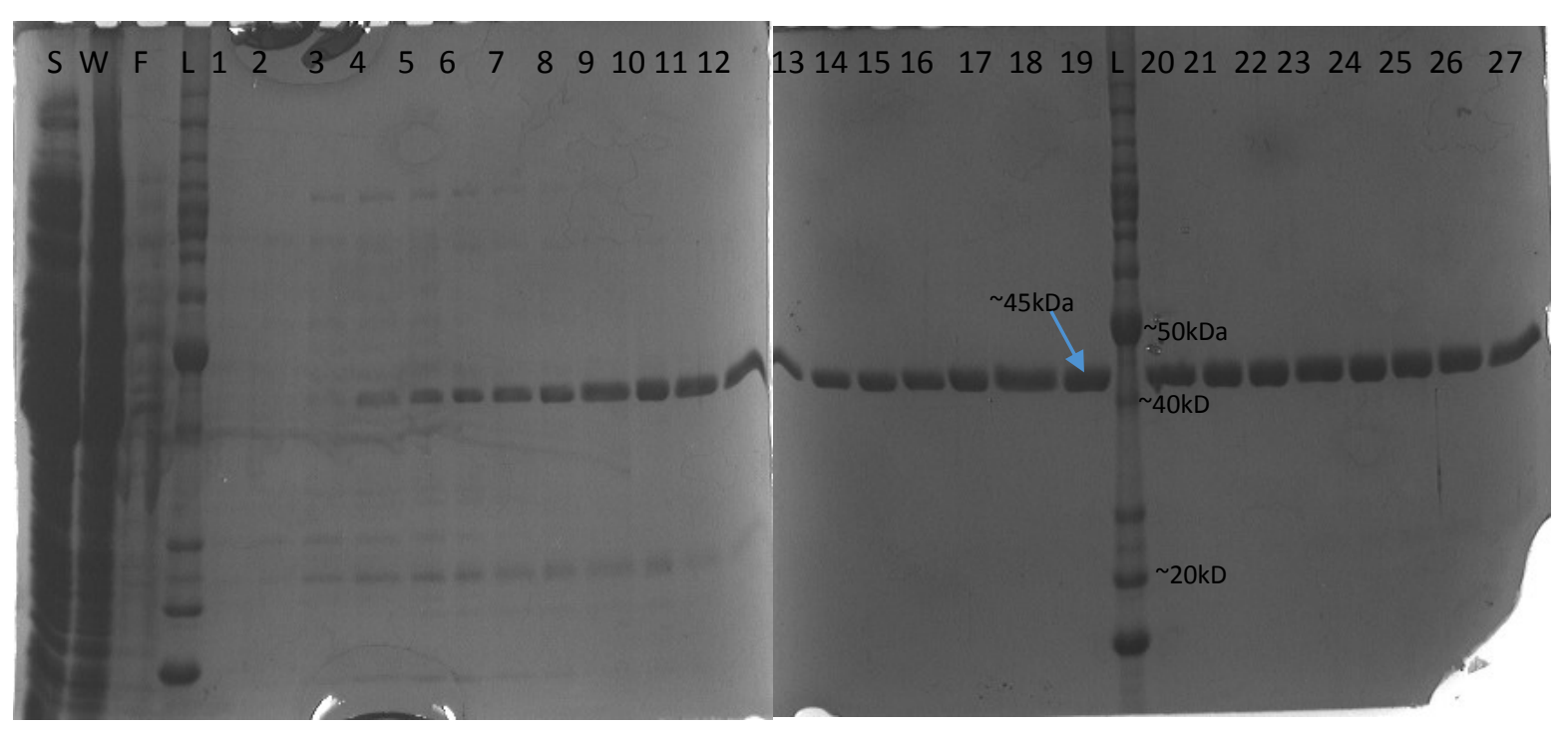

Figure 3.3. SDS-PAGE gels, stained with Comassie blue, of the purification of eCBL-A207S. Lanes are designated as $\mathrm{S}=$ Sample, $\mathrm{W}=$ Wash with buffer $\mathrm{A}, \mathrm{F}=$ Flow through and $\mathrm{L}=$ Ladder. Fractions 13-27 were pooled and concentrated.

Table 3.2. Site-directed variants and their protein yields.

\begin{tabular}{|cc|}
\hline $\begin{array}{l}\text { eCBL } \\
\text { variants }\end{array}$ & Yield (mg/L) \\
\hline G57S & 3.11 \\
\hline A207S & 3.74 \\
\hline $\begin{array}{c}\text { yCGL } \\
\text { variants } \\
\text { S50G }\end{array}$ & Yield $(\mathrm{mg} / \mathrm{L})$ \\
\hline S50A & 5.14 \\
\hline S200A & 2.96 \\
\hline
\end{tabular}




\subsubsection{Kinetic Characterization of the Site-Directed Variants}

The $\mathrm{k}_{\text {cat }} / \mathrm{K}_{\mathrm{m}}$ values of the G57S and A207S variants of eCBL are decreased by 6 -fold, compared to the wild-type enzyme and the change is dominated by an increase of similar magnitude in the $\mathrm{K}_{\mathrm{m}}$ (table 3.2.). The 5.4 and 11 -fold decreases in catalytic efficiency for the S50A and S50G variants of yCGL and 6-fold decrease for yCGL-S200A were influenced by matched 2-4-fold changes in both $\mathrm{k}_{\mathrm{cat}}$ and $\mathrm{K}_{\mathrm{m}}$. Substitution of yCGL-S50 has a modestly smaller effect on turnover than removal of the hydroxyl group of S200 as the $\mathrm{k}_{\mathrm{cat}}$ of the former is reduced by 2.2 and 2.8fold, respectively, while that of S200A is decreased 4.8-fold. 
Table 3.3. Steady state kinetic parameters of wild-type and site-directed variants of yCGL and eCBL.

\begin{tabular}{|c|c|c|c|}
\hline \multicolumn{4}{|c|}{$\boldsymbol{\beta}$-elimination: cystathionine $\rightarrow$ homocysteine + pyruvate $+\mathrm{NH}^{+}$} \\
\hline Enzyme & Kcat $\left(\mathbf{s}^{-1}\right)$ & $\mathbf{K}_{\mathbf{m}}^{\mathrm{L}-\mathrm{Cth}}(\mathbf{m M})$ & $\mathbf{K}_{\mathrm{cat}} / \mathbf{K}_{\mathbf{m}}{ }^{-\mathrm{Cth}}\left(\mathbf{M}^{-1} \mathbf{s}^{-1}\right)$ \\
\hline Wt eCBL & $44 \pm 1$ & $0.14 \pm 0.02$ & $(3.2 \pm 0.3) \times 10^{5}$ \\
\hline $\mathrm{Wt}_{\mathrm{eCBL}}{ }^{b}$ & $34.1 \pm 0.6$ & $0.18 \pm 0.01$ & $(1.9 \pm 0.1) \times 10^{5}$ \\
\hline eCBL G57S & $73.7 \pm 6.2$ & $1.5 \pm 0.4$ & $(5.0 \pm 0.8) \times 10^{4}$ \\
\hline eCBL A207S & $31.3 \pm 1.7$ & $0.6 \pm 0.1$ & $(5.3 \pm 0.8) \times 10^{4}$ \\
\hline \multicolumn{4}{|c|}{$\gamma$-elimination: cystathionine $\rightarrow$ cysteine $+\alpha$-ketobutyrate $+\mathrm{NH}^{+}$} \\
\hline Wt yCGL & $1.96 \pm 0.03$ & $0.77 \pm 0.04$ & $(2.5 \pm 0.1) \times 10^{3}$ \\
\hline Wt yCGL ${ }^{c}$ & $1.51 \pm 0.03$ & $0.71 \pm 0.04$ & $2.1 \pm 0.1 \times 10^{3}$ \\
\hline yCGL S50A & $0.88 \pm 0.02$ & $1.9 \pm 0.1$ & $(4.6 \pm 0.2) \times 10^{2}$ \\
\hline yCGL S50G & $0.71 \pm 0.02$ & $3.2 \pm 0.2$ & $(2.25 \pm 0.06) \times 10^{2}$ \\
\hline yCGL S200A & $0.408 \pm 0.006$ & $1.25 \pm 0.05$ & $(3.3 \pm 0.1) \times 10^{2}$ \\
\hline
\end{tabular}

${ }^{a}$ Kinetic parameters were determined at $25^{\circ} \mathrm{C}$ in $50 \mathrm{mM}$ Tris buffer $(\mathrm{pH} 8.5)$ containing $20 \mu \mathrm{M}$ PLP, $2 \mu \mathrm{M}$ DTNB, 0.004-6.2mM L-Cth and 0.3-1.03 $\mu \mathrm{M}$ or $0.8-0.9 \mu \mathrm{M}$ enzyme for eCBL and yCGL variants, respectively. Data were fit to equations 3.1 and 3.2

${ }^{b}$ Values for kinetic parameters for wt-eCBL are from Lodha et al., 2010

${ }^{c}$ Values for kinetic parameters for wt-yCGL are from Hopwood et al., 2014 


\subsection{Discussion}

The fold-type I enzymes of the transsulfuration pathways (CBL, CGL and CGS) are a good model for investigation of the structure-function relationships that underlie substrate and reaction specificity as they share a common overall fold, but catalyze distinct transformations of similar or identical amino acid substrates (Aitken et al., 2011). The cystathionine-hydrolyzing enzymes CBL and CGL are particularly interesting as they preferentially yield homocysteine and cysteine, respectively. Several of the active site residues of these enzymes are conserved, including the two arginines that bind the distal and $\alpha$-carboxylate moieties of the cystathionine substrate and the tyrosine (eCBL-Y56, yCGL-Y49) and serine (eCBL-S339, yCGL-S334) residues that are proposed to tether and guide the $\varepsilon$-amino group of the active site lysine, following its release from Schiff base linkage with the cofactor (Lodha and Aitken, 2011; Jaworski et al., 2012). Studies from our lab have led to the theory that the $\varepsilon$-amino group of the catalytic base is differentially tethered to restrict its access to $\mathrm{C} \alpha$ of the substrate in eCBL, but to allow it to swing more freely to enable proton transfer between the $\mathrm{C} \beta$ and $\mathrm{C} 4$ ' positions of the substrate and cofactor, respectively in yCGL (Aitken et al., 2011; Manders et al., 2013). This aligns with the earlier observation by Yamagata and coworkers that while the reaction specificity of eCBL is restricted to only $\beta$-elimination, yCGL catalyzes both the $\beta$ and $\gamma$-elimination of their common, pseudosymmetric substrate (Yamagata et al., 1993). However, as the tyrosine and serine residues that tether the lysine are conserved between CBL and CGL sequences, the factor(s) that distinguishes the mobility of the catalytic base, with respect to the substrate-cofactor complex, have not yet been identified. Comparison of the eCBL and yCGL structures and those of eCBL and the eCBL-AVG complex identified two positions, conserved as distinct residues in CBL and 
CGL enzymes and not been previously investigated and that may play a role in regulating the mobility of the active site lysine residue that acts as the catalytic base.

Residue eCBL-G57 and the corresponding yCGL-S50 are immediately adjacent to the tyrosine residue (eCBL-Y56, yCGL-Y49) that, in collaboration with the conserved serine residue (eCBLS339, yCGL-S334), tethers the side chain amino group of the catalytic base. Any effect of this residue would likely be indirect and mediated by influencing the position of the adjacent tyrosine. Farsi and colleagues investigated the role of eCBL-F55 and the corresponding yCGLE48 as determinants of specificity. This position, together with eCBL-G57/yCGL-S50 flanks the tyrosine residue (eCBL-Y56, yCGL-Y49). However, while the catalytic efficiency of the interconverting substitutions eCBL-F55D and yCGL-E48F variants is reduced 74 and 8.8 -fold, respectively, their reaction specificity is not altered, thereby disproving the structure-based suggestion that this residue, together with eCBL-Y338 and the corresponding yCGL-E333, regulate specificity (Farsi et al., 2009; Clausen et al., 1998; Messerschmidt et al., 2003). Similarly, the 5-11-fold reductions in the catalytic efficiency of eCBL-G57S and the S50G and S50A substitutions of yCGL, demonstrate that the residue in this position does not play a primary role in controlling the mobility of the active site lysine or defining a catalytically competent active site conformation. However, it suggests that this residue may act in collaboration with other active site residues to influence reaction specificity.

In contrast with the indirect, tyrosine-mediated influence on the mobility of the catalytic base proposed for yCGL-S50, the position of yCGL-S200A is such that a direct influence was proposed, via interaction with the side chain of the catalytic base, as the corresponding residue is 
within hydrogen-bonding distance of the active site lysine in the eCBL-AVG structure. This residue, positioned centrally behind the aromatic ring of the cofactor, is conserved as alanine in bacterial CBL enzymes and, therefore, unable to interact in hydrogen-bonding interactions via the side chain. The 6-fold reduction in the catalytic efficiency of both the eCBL-A207S and yCGL-S200A variants indicate that the side-chain hydroxyl of the latter likely does not interact with or play a role in modulating the mobility of the catalytic base in the context of the yCGL active site. The observed minor effect on catalytic efficiency for both enzymes may be due to positioning of the PLP as, together with Y103 (eCBL-Y111), which forms $\pi$-stacking interactions with the PLP ring, residues T180 and S200 of yCGL pack against the cofactor to provide a binding pocket (Messerschmidt et al., 2003).

A thorough understanding of the mechanisms that control substrate and reaction specificity is a necessary step to enable the engineering of PLP-dependent enzymes and is essential for the development of novel antimicrobial compounds and therapeutics (Clausen et al., 1997; Ejim et al., 2007). The results of this study suggest that residues S50 and S200 of yCGL and the corresponding G57 and A207 of eCBL play a minor role in ensuring a catalytically competent active site conformation, but do not directly influence the mobility and/or positioning of the catalytic base. Our lab has characterized the roles of many of the residues lining the active site of eCBL, eCGS and yCGL, identifying the individual influences of each (Farsi et al., 2009; Lodha et al., 2010; Lodha and Aitken, 2011; Jaworski et al., 2012; Hopwood et al., 2014). Future studies can now explore the effect of modifying pairs or groups of residues, as initiated in our development of chimeric eCBL-eCGS constructs, on the substrate and reaction specificity of the enzymes of the transsulfuration pathways (Manders et al., 2013). 


\section{Conclusion}

Enzymes catalyzing transformations of amino acids are generally reliant on the catalytically versatile pyridoxal 5'-phosphate (PLP) cofactor. The range of reactions catalyzed by the cofactor is regulated by the protein scaffold of each enzyme and, as such, identification of the factors that regulate substrate and reaction specificity is a goal researchers have pursued and refined, with each new piece of information, for decades. Knowledge of the structure-function relationships that underlie specificity, particularly for enzymes reliant on a cofactor able to catalyze a range of transformations, will enhance the success of protein engineering studies attempting to develop enzymes with novel activities for industrial applications. This is an attractive goal as it can enable enhanced yields, energy efficiency and reduced environmental impact.

The enzymes cystathionine $\gamma$-synthase (CGS) and cystathionine $\beta$-lyase (CBL) of the plant and bacterial transsulfuration pathway and cystathionine $\beta$-synthase (CBS) and cystathionine $\gamma$-lyase (CGL) of the yeast and mammalian reverse transsulfuration pathway interconvert cysteine and homocysteine, the immediate precursor of methionine. The CBL/CGL pair provides an ideal model system to probe the differences in the protein components of PLP-dependent enzymes to explore the structure-function relationships that regulate enzyme activity as they share a common structure and catalyze distinct hydrolysis reactions with a common substrate. Comparison of the structures of Escherichia coli CBL (eCBL) and Saccharomyces cerevisiae CGL (yCGL) identified differences at the dimer interface and in the active site and site-directed variants of six residues in eCBL and 4 in yCGL were constructed and characterized to investigate their influence on the activities of these enzymes. 
The dimer interface of yCGL is characterized by nonpolar interactions, while hydrogen-bonds and salt-bridges between polar and charged residues dominate that of eCBL. Single and double charge-reversal substitutions, targeting the K5-D382 and K17-D37 salt bridges of eCBL, similarly reduced the catalytic efficiency by 11-20-fold, despite the greater effect of the former on the stability of the enzyme. This demonstrates that while even small conformational changes in this region are conveyed to the active site, the distinct dimer interfaces of eCBL and $\mathrm{yCGL}$ are unlikely to be a primary determinant of specificity. Future studies will continue this work, with interconverting substitutions (i.e. replacing charged interactions with the corresponding nonpolar residues of yCGL) and investigation of the dimer interface of yCGL.

A pair of distinct active-site residues were also investigated, via characterization of the S50A, S50G and S200A substitutions of yCGL and the corresponding G57S and A207S of eCBL, to assess their differential effect in the context of each enzyme. The modest 5-11-fold reduction in the catalytic efficiency of these site-directed variants suggests that these residues do not directly influence the positioning of the $\varepsilon$-amino group of the catalytic base and, as such, are unlikely to be determinants of reaction specificity. Our research to date has demonstrated the complexity of the structure-function relationships that underlie reaction specificity in the closely related enzymes of the transsulfuration pathways and show that multiple residues act in concert for this purpose. From the perspective of cellular metabolism, this provides the advantage of stability as while a single mutation would be unlikely to change the products of a pathway central to primary metabolism, specificity can be changed via a gradual evolutionary process, requiring multiple, successive substitutions, as demonstrated by the members of the $\gamma$-subfamily of fold-type I of PLP-dependent enzymes. Our work has shown that the positioning of the substrate with respect 
to the catalytic base is a primary factor regulating specificity and has identified many of the residues responsible for this (Farsi et al., 2009; Lodha et al., 2010; Lodha and Aitken, 2011; Aitken et al., 2011; Jaworski et al., 2012; Manders et al., 2014). Future studies will explore both the role of second-shell residues (i.e. those in contact with active-site residues) in the differential positioning of active-site residues conserved in both enzymes as well as the effect of combining substitutions of multiple active-site and/or second-shell residues identified as distinct between the active sites of eCBL and yCGL with the goal of refining our understanding of the mechanism(s) underlying reaction specificity. 


\section{References}

Aitken, S. M., and Kirsch, J. F. 2005. The enzymology of cystathionine biosynthesis: Strategies for the control of substrate and reaction specificity. Archives of Biochemistry and Biophysics, 433(1), 166-175.

Aitken, S. M., Lodha, P. H., and Morneau, D. J. K. 2011. The enzymes of the transsulfuration pathways: Active-site characterizations. Biochimica et Biophysica Acta-Proteins and Proteomics, 1814(11), 1511-1517.

Ardo, Y. 2006. Flavour formation by amino acid catabolism. Biotechnology Advances, 24, 238242.

Agarwal, P. K., Schultz, C., Kalivretenos, A., Ghosh, B., and Broedel Jr, S. E. 2012. Engineering a hyper-catalytic enzyme by photoactivated conformation modulation. The Journal of Physical Chemistry Letters, 3(9), 1142-1146.

Belfaiza, J., Parsot, C., Martel, A., De La Tour, C. B., Margarita, D., Cohen, G. N., and SaintGirons, I. 1986. Evolution in biosynthetic pathways: two enzymes catalyzing consecutive steps in methionine biosynthesis originate from a common ancestor and possess a similar regulatory region. Proceedings of the National Academy of Sciences, 83(4), 867-871.

Brosnan, J. T., and Brosnan, M. E. 2006. The sulfur-containing amino acids: An overview. Journal of Nutrition, 136(6), 1636S-1640S.

Brown, E. D., and Wright, G. D. 2005. New targets and screening approaches in antimicrobial drug discovery. Chemical reviews, 105(2), 759-774. 
Carter P.J. 2011. Introduction to current and future protein therapeutics: A protein engineering perspective. Exp Cell Res. 317, 1261-1269.

Christen, P., and Mehta, P. K. 2001. From cofactor to enzymes. The molecular evolution of pyridoxal-5'-phosphate-dependent enzymes. The Chemical Record, 1(6), 436-447.

Clausen, T., Huber R., Laber B., Pohlenz H.D., and Messerschmidt A. 1996. Crystal structure of the pyridoxaL-5'-phosphate dependent cystathionine $\beta$ - lyase from Escherichia coli at $1.83 \AA$. $J$ Mol.Bio. 262, 202-224.

Clausen, T., Laber B., and Messerschmidt A. 1997. Mode of action of cystathionine Beta-Lyase. Bio.Chem. 378, 321-326.

Clausen, T., Huber, R., Prade, L., Wahl, M. C., and Messerschmidt, A. 1998. Crystal structure of Escherichia coli cystathionine $\gamma$-synthase at $1.5 \AA$ resolution. The EMBO Journal, 77(23), 68276838.

Dobric, N., Limsowtin, G. K., Hillier, A. J., Dudman, N. P., and Davidson, B. E. 2000. Identification and characterization of a cystathionine $\beta / \gamma$-lyase from Lactococcus lactis ssp. cremoris MG1363. FEMS microbiology letters, 182(2), 249-254.

Ejim L.J., Blanchard J.E., Koteva K.P., Sumerfield R., Elowe N.H., Chechetto J.D., Brown E.D., Junop M.S., and Wright G.D. 2007. Inhibitors of bacterial cystathionine $\beta$-lyase: leads for new antimicrobial agents and probes of enzyme structure and function. J Med Chem 50, 755-764.

Eliot, A. C., and Kirsch, J. F. 2004. Pyridoxal phosphate enzymes: mechanistic, structural, and evolutionary considerations. Annual review of biochemistry, 73(1), 383-415. 
Ellman, G. L. 1959. Tissue sulfhydryl groups. Archives of biochemistry and biophysics, 82(1), 70-77.

Farsi A., Lodha P.H., Skanes J.E., Los H., Kalidindi N., and Aitken S.M. 2009. Interconversion of a pair of active-site residues in Escherichia coli cystathionine $\gamma$-synthase, E. coli cystathionine $\beta$-lyase, and Saccharomyces cereisiae cystathionine $\gamma$ - lyase and development of tools for the investigation of their mechanisms and reaction specificity. Biochem. Cell. Biol. 87, 445-457.

Hopwood, E. M., Ahmed, D., and Aitken, S. M. 2014. A role for glutamate-333 of Saccharomyces cerevisiae cystathionine $\gamma$-lyase as a determinant of specificity. Biochimica et Biophysica Acta (BBA)-Proteins and Proteomics, 1844(2), 465-472.

Hult, K., and Berglund, P. 2007. Enzyme promiscuity: mechanism and applications. Trends in biotechnology, 25(5), 231-238.

Jansonius, J.N. 1998. Structure, evolution and action of vitamin B6-dependent enzymes. Current Opinion in Structural Biology 8, 759-769.

Jaworski, A. F., Lodha, P. H., Manders, A. L., and Aitken, S. M. 2012. Exploration of the active site of Escherichia coli cystathionine $\gamma$-synthase. Protein Science, 21(11), 1662-1671.

Jaworski, A.F and Aitken, S.M 2013. Exploration of the Six Tryptophan Residues of Escherichia coli cystathionine $\beta$-lyase as Probes of Enzyme Conformational Change. Archives of Biochemistry and Biophysics, 538, 138-144.

Keleş, S., \& Bilgen, S. 2012. Renewable energy sources in Turkey for climate change mitigation and energy sustainability. Renewable and Sustainable Energy Reviews, 16(7), 5199-5206. 
Laber, B., Clausen, T., Huber, R., Messerschmidt, A., Egner, U., Muller-Fahrnow, A., Pohlenz, H.D. 1995. Cloning, Purification, and Crystallization of Escherichia coli Cystathionine $\beta$-lyase. FEBS, 379, 94-96.

Lehmann, M., and Wyss, M. 2001. Engineering proteins for thermostability: the use of sequence alignments versus rational design and directed evolution. Current Opinion in Biotechnology, 12(4), 371-375.

Liu, M., Nauta, A., Francke, C., and Siezen, R. J. 2008. Comparative genomics of enzymes in flavor-forming pathways from amino acids in lactic acid bacteria. Applied and environmental microbiology, 74(15), 4590-4600.

Lodha, P.H., Jaworski, A.F., and Aitken, S.M. 2009. Characterization of site-directed mutans of residues R58, R59, D116, W340 and R372 in the active site of E.Coli cystathionine $\beta$-lyase. Protein Science, 19, 383-391.

Lodha, P.H., Jaworski, A.F., and Aitken, S.M. 2010. Characterization of site-directed mutants of residues R58, R59, D116, W340 and R372 in the active site of E. coli cystathionine ß-lyase. Protein Science, 19(3), 383-391.

Lodha, P.H and Aitken, S.M. 2011. Characterization of the Side-Chain Hydroxyl Moieties of Residues Y56, Y111, Y238, Y338, and S339 as Determinants of Specificity in E. coli Cystathionine $\beta$-Lyase. Biochemsitry, 50, 9876-9885.

Manders, A.L., Jaworski, A.F., Ahmed, M., and Aitken, S.M. 2013. Exploration of structurefunction relationships in Escherichia coli cystathionine $\gamma$-synthase and cystathionine $\beta$-lyase via chimeric constructs and site-specific substitutions. Biochimica et Biophysica Acta (BBA)Proteins and Proteomics, 1834(6), 1044-1053. 
Messerschmidt, A., Worbs, M., Steegborn, C., Wahl, M.C., Huber, R., Laber, B., and Clausen, T. 2003. Determinants of enzymatic specificity in the Cys-Met-metabolism PLP-dependent enzymes family: crystal structure of cystathionine $\gamma$-lyase from yeast and intrafamiliar structure comparison. Biol. Chem., 384, 373-386.

Ordu, E. and Karagüler, N.G. 2012. Protein engineering applications on industrially important enzymes: Candida methylica FDH as a case study. INTECH Open Access Publisher.

Percudani, R. and Peracchi, A. 2003. A genomic overview of pyridoxal -phosphate-dependent enzymes. EMBO Reports, 4, 850-854.

Schneider, G., Kack, H., and Lindqzist, Y. 2000. The manifold of vitamin B6 dependent enzymes. Structure, 8, R1-R6.

Steegborn, C., Clausen, T., Sondermann, P., Jacob, U., Worbs, M., Marinkovic, S., and Wahl, M. C. 1999. Kinetics and inhibition of recombinant human cystathionine $\gamma$-lyase toward the rational control of transsulfuration. Journal of Biological Chemistry, 274(18), 12675-12684.

Stipanuk, M.H. 2004. Sulfur amino acid metabolism: Pathways for production and removal of homocysteine and cysteine.

Toney M.D. 2004. Reaction specificity in pyridoxal phosphate enzymes. Biochimica et Biophysica Acta Biochemistry and Biophysics, 433 (2005), 279-287.

Vogel, H.J., and Bonner, D.M. 1956. Acetylornithinase of Escherichia coli: partial purification and some properties. Journal of Biological Chemistry, 218(1), 97-106. 
Walters, J., Milam, S.L., and Clark, A.C. 2009. Practical approaches to protein folding and assembly: spectroscopic strategies in thermodynamics and kinetics. Methods in enzymology, 455, 1-39.

Woycechowsky, K.J., and Hilvert, D. 2006. Novel enzymes through design and evolution. Advances in Enzymology and Related Areas of Molecular Biology, Protein Evolution, 75, 241.

Yamagata, S., D'andrea, R. J., Fujisaki, S., Isaji, M., and Nakamura, K. 1993. Cloning and bacterial expression of the CYS3 gene encoding cystathionine gamma-lyase of Saccharomyces. $J$ Bacteriol, 175(15), 4800-4808. 